\title{
Constructal theory of global circulation and climate
}

\author{
A. Heitor Reis ${ }^{\mathrm{a}, *}$, Adrian Bejan ${ }^{\mathrm{b}}$ \\ ${ }^{a}$ University of Évora, Department of Physics and Geophysics Center of Évora, Colégio Luis Verney, Rua Romão Ramalho, \\ 59, 7000-671 Evora, Portugal \\ ${ }^{\mathrm{b}}$ Duke University, Department of Mechanical Engineering and Materials Science, Durham, NC 27708-0300, USA
}

Received 20 November 2004

Available online 19 January 2006

\begin{abstract}
The constructal law states that every flow system evolves in time so that it develops the flow architecture that maximizes flow access under the constraints posed to the flow. Earlier applications of the constructal law recommended it as a self-standing law that is distinct from the second law of thermodynamics. In this paper, we develop a model of heat transport on the earth surface that accounts for the solar and terrestrial radiation as the heat source and heat sink and with natural convection loops as the transport mechanism. In the first part of the paper, the constructal law is invoked to optimize the latitude of the boundary between the Hadley and the Ferrel cells, and the boundary between the Ferrel and the Polar cells. The average temperature of the earth surface, the convective conductance in the horizontal direction as well as other parameters defining the latitudinal circulation also match the observed values. In the second part of the paper, the constructal law is invoked in the analysis of atmospheric circulation at the diurnal scale. Here the heat transport is optimized against the Ekman number. Even though this second optimization is based on very different variables than in the first part of the paper, it produces practically the same results for the earth surface temperature and the other variables. The earth averaged temperature difference between day and night was found to be approximately $7 \mathrm{~K}$, which matches the observed value. The accumulation of coincidences between theoretical predictions and natural flow configuration adds weight to the claim that the constructal law is a law of nature.
\end{abstract} (c) 2005 Elsevier Ltd. All rights reserved.

Keywords: Constructal theory; Thermodynamics; Non-equilibrium; Convection; Radiation; Global circulation; Climate

\section{Objectives}

Thermodynamics has reached an impasse similar to the development of the heat engine two centuries ago. The real world (nature, physics) has structure, organization, pattern. Until now, thermodynamics was not concerned with the architecture (the drawings) of the systems that inhabit its black boxes. It should have been.

The tissues of energy flow systems like the fabric of society and all the tissues of biology, are optimized architectures. Not just "any" architectures, as in the black boxes of classical thermodynamics, but the optimal, or the nearoptimal architectures. The climbing to this high podium

\footnotetext{
* Corresponding author.

E-mail address: ahr@uevora.pt (A.H. Reis).
}

of performance is the transdisciplinary effort - the balance between seemingly unrelated flows, territories, and disciplines. No flow system is an island. No river exists without its wet plain. No city thrives without its farmland and open spaces. Everything that flowed and lived to this day to "survive" is in an optimal balance with the flows that surround it and sustain it. This balancing act - the optimal distribution of imperfection - generates the very design of the process, power plant, city, geography and economics.

In this paper we examine theoretically the largest flow system on earth from the point of view of the constructal theory of organization in nature [1,2], which now constitutes the thermodynamics of non-equilibrium (flow) systems with configuration $[3,4]$. The new development is that there are two time arrows in physics, not one. The old is the time arrow of the second law of thermodynamics, the arrow of irreversibility: every thing flows from high to low. The new 


\begin{tabular}{|c|c|c|c|}
\hline \multicolumn{4}{|c|}{ Nomenclature } \\
\hline$A$ & area, $\mathrm{m}^{2}$ & $U$ & earth rotation speed averaged along the merid \\
\hline$B$ & constant, Eq. (11) & & ian, $\mathrm{m} \mathrm{s}^{-1}$ \\
\hline$c_{p}$ & specific heat capacity at constant pressure, & $U_{0}$ & earth rotation speed at the equator, $\mathrm{m} \mathrm{s}^{-1}$ \\
\hline$C_{3 / 2}$ & $\begin{array}{l}\mathrm{J} \mathrm{Kg} \\
\text { thermal conductance, } \mathrm{K}^{5 / 2}\end{array}$ & $\begin{array}{l}u \\
u^{*}\end{array}$ & $\begin{array}{l}\text { meridional velocity component, } \mathrm{m} \mathrm{s}^{-1} \\
\text { friction velocity, } \mathrm{m} \mathrm{s}^{-1}\end{array}$ \\
\hline$D$ & $\begin{array}{l}\text { convective conductance in the horizontal direc- } \\
\text { tion, } \mathrm{W} \mathrm{m}^{-2} \mathrm{~K}^{-1}\end{array}$ & $x$ & $\begin{array}{l}\text { fraction of the earth's surface acting as a hea } \\
\text { source }\end{array}$ \\
\hline$E k$ & Ekman number & $W$ & width, $\mathrm{m}$ \\
\hline & earth-sun view factor & $w$ & power dissipated by fluid friction on the earth's \\
\hline \multicolumn{4}{|c|}{$C_{D}, C_{0}, C_{1}$ constants } \\
\hline$g$ & gravitational acceleration, $\mathrm{m} \mathrm{s}^{-2}$ & & \\
\hline$H$ & height of the friction layer, $\mathrm{m}$ & \multicolumn{2}{|c|}{ Greek symbols } \\
\hline$k$ & von Karman constant, Eq. (35) & $\alpha$ & albedo of the earth \\
\hline$L$ & length, $\mathrm{m}$ & $\beta$ & coefficient of volumetric thermal expansion, $\mathrm{K}^{-}$ \\
\hline$L_{\mathrm{O}}$ & Obukhov length, $\mathrm{m}$ & $\varepsilon_{\mathrm{M}}$ & eddy diffusivity for momentum, $\mathrm{m}^{2} \mathrm{~s}^{-1}$ \\
\hline$P$ & pressure, $\mathrm{Pa}$ & $\varepsilon_{\mathrm{H}}$ & eddy diffusivity for heat, $\mathrm{m}^{2} \mathrm{~s}^{-1}$ \\
\hline$q$ & heat transfer rate, $\mathrm{W}$ & $\gamma$ & earth greenhouse factor \\
\hline$q^{\prime \prime}$ & heat flux, $\mathrm{W} \mathrm{m}^{-2}$ & $\Lambda$ & ratio between total enthalpy and sensible heat in \\
\hline$R$ & earth radius, $\mathrm{m}$ & & the atmosphere \\
\hline$R_{\mathrm{g}}$ & gas constant, $\mathrm{J} \mathrm{kg}^{-1} \mathrm{~K}^{-1}$ & $\sigma$ & Stefan-Boltzmann constant \\
\hline & overall entropy generation on earth, $\mathrm{W} \mathrm{K}^{-1}$ & $\theta$ & latitude \\
\hline$S_{0}$ & solar constant, $1380 \mathrm{~W} \mathrm{~m}^{-2}$ & $\xi$ & non-dimensional factor, Eq. (12) \\
\hline$T$ & temperature, $\mathrm{K}$ & & \\
\hline
\end{tabular}

is the time arrow of the constructal law, the arrow of how every flowing thing acquires architecture. The "how" is condensed in the constructal law: existing configurations assure their survival by morphing in time toward easier flowing configurations. The constructal time arrow unites physics with biology and engineering (after all, engineering is the biology and medicine of "man + machine species").

We see this union in Fig. 1, which expresses the vision proposed in Fig. 3.16 of Ref. [1] and earlier. The earth with its solar heat input, heat rejection, and wheels of atmospheric and oceanic circulation, is a heat engine without shaft: its maximized (but not ideal) mechanical power output cannot be delivered to an extraterrestrial system. Instead, the earth engine is destined to dissipate through air and water friction and other irreversibilities (e.g., heat leaks across finite $\Delta T$ ) all the mechanical power that it produces. It does so by "spinning in its brake" the fastest that it can (hence the winds and the ocean currents, which proceed along easiest routes). Because the flowing earth is a constructal heat engine, its flow configuration has evolved in such a way that it is the least imperfect that it can be. It produces maximum power, which it then dissipates at maximum rate. As we show later in this section, maximum dissipation has been invoked ad hoc in geophysics: all such discussions refers only to what goes on in the brake.

The heat engines of engineering and biology (power plants, animal motors) have shafts, rods, legs and wings that deliver the mechanical power to external entities that use the power (e.g., vehicles and animal bodies needing propulsion). Because the engines of engineering and biology are constructal, they morph in time toward flow configurations that make them the least imperfect that they can be. Therefore they evolve toward producing maximum mechanical power (under finiteness constraints), which, for them, means a time evolution toward minimum dissipation (minimum entropy generation rate).

On the outside an engineering or biology engine, all the mechanical power that the engine delivers is destroyed through friction and other irreversibility mechanisms (e.g., transportation and manufacturing for man, animal locomotion and body heat loss to ambient). The engine and its immediate environment (the brake), as one thermodynamic system, are analogous to the whole earth (Fig. 1). When all is said and done, the flowing earth (with all its engine + brake components, rivers, fish, turbulent eddies, etc.) mixes the earth's crust most effectively-more effectively than in the absence of constructal phenomena of generation of flow configuration. Dry river basins, Egyptian pyramids and junk yards are fossils of the constructal flow systems that once thrived.

Another objective of this paper is to establish a common theoretical basis for two distinct domains: the climate models that have been developed ad hoc in geophysics, and the heat engine models that have been developed ad hoc for wind power calculations in thermodynamics. Both domains are very active, voluminous, and established - to review them is not the objective here. The common basis is provided by constructal theory $[1,2]$, which is the thought that 


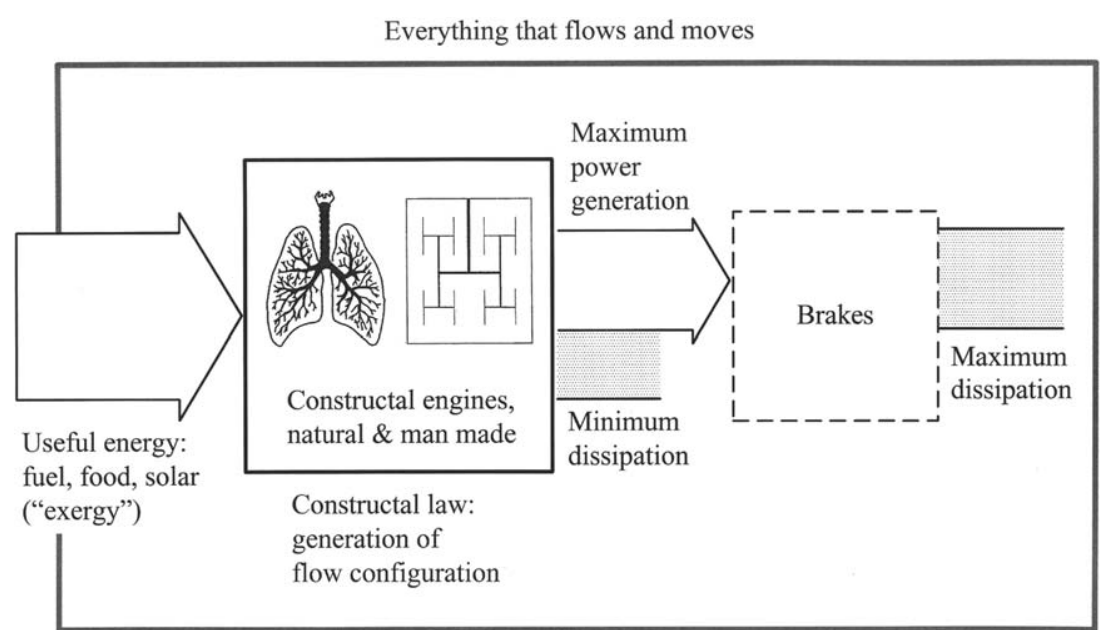

Fig. 1. Every non-equilibrium (flow) component of the earth functions as an engine that drives a brake. The constructal law governs 'how' the system functions: by generating a flow architecture that distributes imperfections optimally to fill the flow space. The 'engine' part evolves in time toward generating maximum power (or minimum dissipation) and, as a consequence, the 'brake' part exhibits maximum dissipation. Evolution means that each flow system assures its persistence (survival) in time by freely morphing into easier and easier flow structures under finiteness constraints. The arrows proceed from left to right because this is the general drawing for a flow (non-equilibrium) system, in steady or unsteady state. When equilibrium is reached, all the flows cease, and the arrows disappear.

thermodynamic systems far from equilibrium (flow systems) construct flow architectures that provide maximum flow access, or maximum global system performance subject to global constraints. This theoretical basis eliminates the "ad hoc" from both fields, and shows that a single principle rules all the model optimizations that have been demonstrated so far.

This connection is important because atmospheric and oceanic circulation is the largest flow system on earth, and because the implications of the theoretical success demonstrated in the two domains are great. In geophysics, the invocation of a principle of maximization of entropy generation rate has been described as a "law of maximum entropy production which follows deductively from the second law of thermodynamics" [5]. This cannot be true - there is nothing in the second law that says that a steady-state flow system must flow such that its rate of entropy generation is maximum. Furthermore, if that were the case, the principle invoked in geophysics is at best a theorem, not a new law. More recent work on constructal theory [3] shows that an optimization principle of this type is indeed a self-standing law. The emergence of globally optimized flow architectures in all classes of flow systems (animate, inanimate, engineered) is a universal phenomenon. We return to this observation in Section 5.

Climate models based on the maximization of entropy generation rate began with Malkus' [6] hypothesis that in Bénard convection the overall heat transfer rate is maximized through the selection of flow pattern. Examples and reviews are available in [7-13]. The connection between this hypothesis and constructal theory was made in Nelson and Bejan [14], where the constructal law delivered all the known characteristics of Bénard flow and heat transfer, in fluids as well as fluid-saturated porous media.
In this paper, constructal theory is extended to the problem of atmospheric and oceanic circulation driven by heating from the sun. Climate means the average thermohydrodynamic conditions that prevail over a significant period (generally 30 years) at a particular region of earth's surface. Due to non-uniform heating, flows develop on the earth's surface carrying heat from hot to cold regions. Atmospheric and oceanic circulations of a wide range of magnitudes participate in this transfer. Coupling between different scales of heat and mass flows is highly non-linear, therefore making prediction of the thermo-hydrodynamic state of the atmosphere a very hard task $[15,16]$.

Thermodynamically, the earth as a whole is a non-equilibrium closed system - a flow system with heat input and heat rejection, and with internal flows. It is not an isolated thermodynamic system. Furthermore, the earth thermodynamic system is not in a steady state, as can be concluded from the observational evidence that temperature and pressure have spatial and temporal variations. However, if we consider local values averaged over a long period, temperature and pressure become time independent even if their spatial variation is preserved. This procedure implies loss of information of short-period phenomena, but it allows the construction of useful steady-state models of the whole system. These models capture the long-term performance of the earth system and allow useful predictions about climate.

Atmospheric and oceanic circulations are the largest flow structures on earth. Modeling of such flow structures relies on deterministic equations, e.g., conservation of mass, energy, angular momentum and momentum, some of which are non-linear and give rise to additional terms that result from the averaging procedure. The closure of such system of equations is not easy and is achieved with the help of empirical information. In spite of the large 
number of parameters used, such models have shed light on global circulation and climate [17], and have served as reminders that theory is needed.

A different approach is made possible by constructal theory $[1,2]$. Although constructal theory also uses constitutive equations, like mass and energy conservation, it derives the actual flow field (the flow architecture) from the maximization of the flow access performance of the whole system under the existing constraints. The non-uniform heating of the earth's surface and atmosphere drives the earth circulation. According to constructal theory, the purpose of the circulation (the objective of any flow with configuration) is to provide maximum access to the currents that flow, in this case to transfer heat from the equatorial zone to the polar caps. The zones and caps are organized in such a way that perform this transport by the most efficient way, which is the one that maximizes the heat flow or, alternatively, by the flow structure that minimizes the resistance to the global heat flow.

It is instructive to start with a brief review of the results of numerical experiments on global circulation presented in three recent papers [18-20]. Later, we will compare these results with the application of the constructal theory to a simple model general circulation and climate. It is generally accepted that the main forcing factors of the earth global circulation are the differential heating between the equator and the poles, and the rotation rate of the earth. Atmospheric global circulation models (GCMs) have been widely employed to perform numerical experiments of sensitivity of global circulation to these forcing factors.

Navarra and Boccaletti [18], used ECHAM4 (a very recent GCM of the European Centre for Medium Range Weather Forecast (ECMRWF), Max-Planck Institute for Meteorology, Hamburg), to investigate the sensitivity of global circulation to rotation rate. The model was run with day rotation periods of $18,24,72,144$ and $360 \mathrm{~h}$. The results of the meridional streamfunction $\left(\mathrm{kg} \mathrm{s}^{-1}\right)$ and of the averaged zonal velocity component $\left(\mathrm{m} \mathrm{s}^{-1}\right)$ show that if the daily rotation period is $360 \mathrm{~h}$ or $144 \mathrm{~h}$, a single Hadley cell develops from the equator to each pole. If the daily rotation lasts $72 \mathrm{~h}$, the Ferrel cell becomes evident, while at a rotation periods of $24 \mathrm{~h}$ and $18 \mathrm{~h}$ the Polar cell forms and joins the other two. It seems evident that if the earth's rotation rate determines the meridional cells, then it must play an important role in global circulation and climate. In fact, at the latitudes where air rises, atmospheric instability develops and generates strong precipitation. These latitudes correspond to the equatorial zone and to the boundaries between the Polar and the Ferrel cells $\left(\sim 50^{\circ}\right.$ latitude in each hemisphere). On the other hand, at the latitudes between the Hadley and the Ferrel cells, air moves down and hence is compressed adiabatically and heated up. Consequently, clouds and precipitation are almost absent at these latitudes, which correspond to the belt of deserts that occur close $30^{\circ}$ latitude in both hemispheres. This is why the meridional circulation cells are so important in the earth's climate.
The influence of short daily rotation periods upon the atmospheric global circulation was investigated by Jenkins [19] based on a similar model (CCM1), for rotation periods of $22,20,18,16$, and $14 \mathrm{~h}$. The resulting latitude-pressure distributions of the stream function show that the latitudinal positioning of Hadley, Ferrel and Polar cells is practically insensitive to the rotation rate. This is important, because if the rotation period were the main cause of the existence of the three-cell regime of earth's atmospheric general circulation, we would expect this influence to extend noticeably to the latitudinal cell distribution that corresponds to shorter rotation periods.

To clarify this point, consider now a study [20] concerning the effect of differential heating i.e., the effect of varying the difference between the polar and equatorial radiative equilibrium temperatures, $\Delta T_{\mathrm{R}}$. This study used a modified version of ECHAM to incorporate the effect of differential heating, but kept day rotation period constant and equal to $24 \mathrm{~h}$. Their results show that for $\Delta T_{\mathrm{R}}=$ $20^{\circ} \mathrm{C}$, only one Hadley cell develops in each hemisphere, even though the daily rotation period is $24 \mathrm{~h}$. In Figs. 2 and 3 we see the development of three cells at the same rotation period. For $\Delta T_{\mathrm{R}}=30^{\circ} \mathrm{C}$, a weak Ferrel cell develops between the latitudes $30^{\circ}$ and $60^{\circ}$. The case shown in Fig. $4 \mathrm{c}$ corresponds to $\Delta T_{\mathrm{R}}=60^{\circ} \mathrm{C}$ and represents the case of the real atmosphere of the earth. At higher temperature differences $\Delta T_{\mathrm{R}} \sim 130-190^{\circ} \mathrm{C}$, the Polar cell disappears. Then, how do we understand this in light of the currently accepted idea that the current three cells (Hadley, Ferrel and Polar) exist as a consequence of the earth's rotation? Moreover, what role does the temperature differences $\Delta T_{\mathrm{R}}$ play in the development of these cells?

\section{Radiative and convective model}

In accordance with constructal theory, we do not "assume" an equator to pole temperature difference. This difference will come from the deduced three-cell partitioning of atmospheric meridional circulation, and will be a result of the invocation of the constructal law.

The method consists of viewing the sun-earth-universe assembly as an extraterrestrial power plant the power output of which is used for the purpose of forcing the atmosphere and hydrosphere to flow. The power plant models that have been proposed and optimized are listed chronologically in Refs. [21-25]. A spaceship, or the earth, may be viewed as a closed system having two surfaces, a hot surface of area $A_{\mathrm{H}}$ and temperature $T_{\mathrm{H}}$, which is heated by the sun, and a cold surface $\left(A_{\mathrm{L}}, T_{\mathrm{L}}\right)$ cooled by radiation to the universe. On a spaceship, the collector $\left(A_{\mathrm{H}}\right)$ and radiator $\left(A_{\mathrm{L}}\right)$ are the object of design [21]. In the modeling of wind generation on earth, the surfaces $A_{\mathrm{H}}$ and $A_{\mathrm{L}}$ represent the daily illuminated and dark hemispheres [22-24], or the time-averaged equatorial and polar zones $[24,25]$. In all cases, the total radiation heat transfer surface is fixed, 

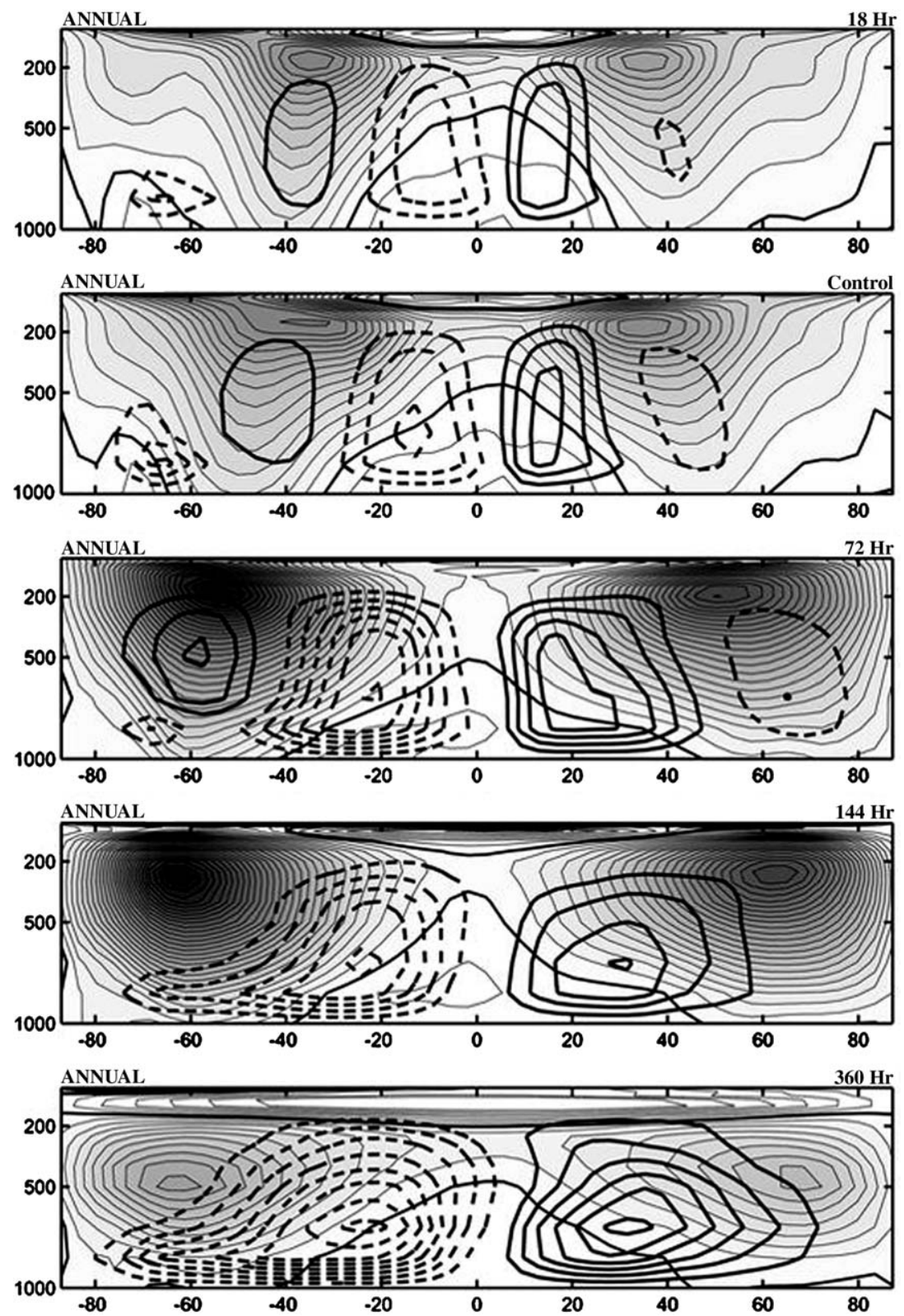

Fig. 2. Thick line: latitude pressure cross section of the meridional stream function (the contour interval is $21,010 \mathrm{~kg} \mathrm{~s}^{-1}$ and $41,010 \mathrm{~kg} \mathrm{~s}{ }^{-1}$ for the bottom two panels). Thin line: zonally averaged zonal speed. From top to bottom: 18, 24, 72, 144, $360 \mathrm{~h}$ a day rotation period (reprinted from [18] with permission).

$A_{\mathrm{H}}+A_{\mathrm{L}}=A$

Global finiteness constraints of this type play a central role in constructal theory. In all the power plant models [21-25] it was found that the power output can be maximized by selecting two parameters, the area fraction $\left(A_{\mathrm{H}} / A\right)$, and the position of the power plant on the thermodynamic temperature scale, for example, the temperature $T_{\mathrm{H}}$.

The field of engineering thermodynamics is unaware of the use of similar principles in geophysics. At the same time, geophysics continues to be unaware of the engineering thermodynamics literature. To bring these two research worlds together is one of the objectives of this paper. To begin with, none of the power plant models dedicated to the generation of wind on earth [21-25] have accounted for buoyancy driven convection - the mechanism by which the generated power is dissipated into heat by the movement of the atmosphere and the oceans. The mechanism has been recognized before, not only in the modeling of atmospheric dynamics, but also in the description of natural convection itself. See, for example, p. 111 in Ref. [26]: "... the convection loop is equivalent to the cycle executed by the working fluid in a heat engine. In principle, this heat engine cycle should be capable of delivering useful work if we insert a propeller in the stream: this is the origin of the "wind power" discussed nowadays in connection with the harnessing of solar work indirectly from the atmospheric heat engine loop. In the absence of work-collecting devices, 

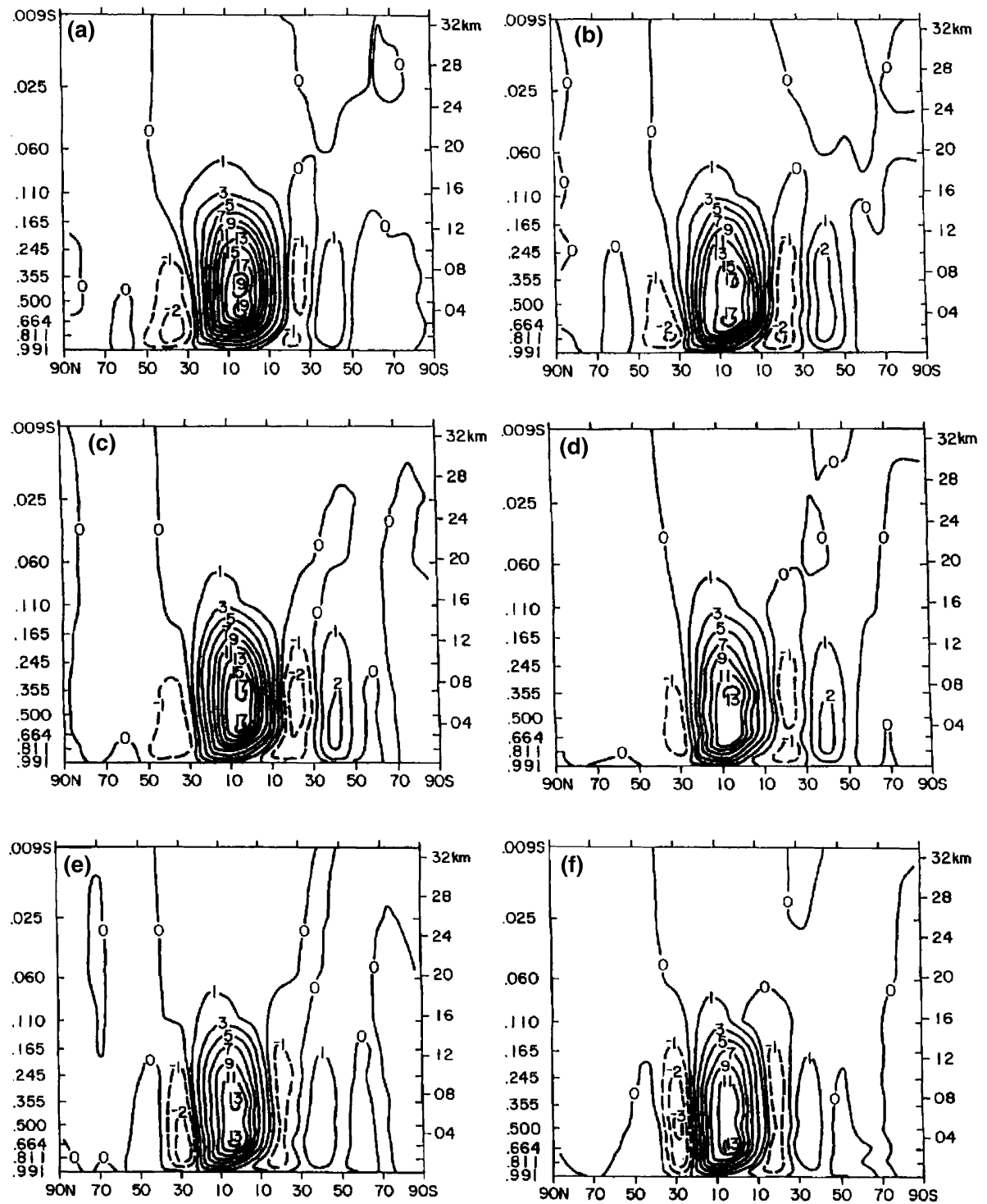

Fig. 3. Zonally averaged mean meridional stream function: (a) control, (b) $22 H R$, (c) $20 H R$, (d) $18 H R$, (e) $16 H R$ and (f) $14 H R$. Units in kg s ${ }^{-1}$ (reprinted from [19] with permission).

the heat engine cycle drives its working fluid fast enough so that its entire work output potential is dissipated by friction in the brake at the interface between what moves and what does not move".

The main features of the earth engine + brake model are illustrated in Figs. 5 and 6. A simpler version was analyzed in Ref. [27]. The surface temperature is time independent. It is averaged over the daily and annual cycles, and is represented by two temperatures $\left(T_{\mathrm{H}}, T_{\mathrm{L}}\right)$ that correspond to the equatorial and polar zones $\left(A_{\mathrm{H}}, A_{\mathrm{L}}\right)$. This radiation model is the same as in Refs. [24,25]. The equatorial surface receives the solar heat current

$q_{\mathrm{sH}}=A_{\mathrm{Hp}}(1-\alpha) f \sigma T_{\mathrm{s}}^{4}$

and radiates into space the heat current

$q_{\mathrm{H}}=A_{\mathrm{H}}(1-\gamma) \sigma T_{\mathrm{H}}^{4}$
Here $T_{\mathrm{s}}, \sigma, \mathrm{f}$ and $\alpha$ are the temperature of the sun as a black body $(5762 \mathrm{~K})$, the Stefan-Boltzmann constant $(5.67 \times$ $\left.10^{-8} \mathrm{~W} \mathrm{~m}^{-2} \mathrm{~K}^{-4}\right)$, the earth-sun view factor $\left(2.16 \times 10^{-5}\right)$, and the albedo of the earth $(0.35)$. Furthermore, $\gamma=0.4$ is the earth's greenhouse factor, or the reflectance in the infrared region [23]. The area $A_{\mathrm{Hp}}$ is the area $A_{\mathrm{H}}$ projected on a plane perpendicular to the direction earth-sun. The ratio $A_{\mathrm{Hp}} / A_{\mathrm{H}}$ varies with the latitude $\theta$ as

$A_{\mathrm{Hp}} / A_{\mathrm{H}}=\frac{\theta+\sin \theta \cos \theta}{2 \pi \sin \theta}$

It decreases from $1 / \pi$, where $A_{\mathrm{H}}$ is a narrow belt along the equator, to $1 / 4$ where $A_{\mathrm{H}}$ covers the globe almost completely (see Fig. 5).

The difference between $q_{\mathrm{sH}}$ and $q_{\mathrm{H}}$ is convected over the earth's surface, from $A_{\mathrm{H}}$ to $A_{\mathrm{L}}$,

$q=q_{\mathrm{sH}}-q_{\mathrm{H}}$ 

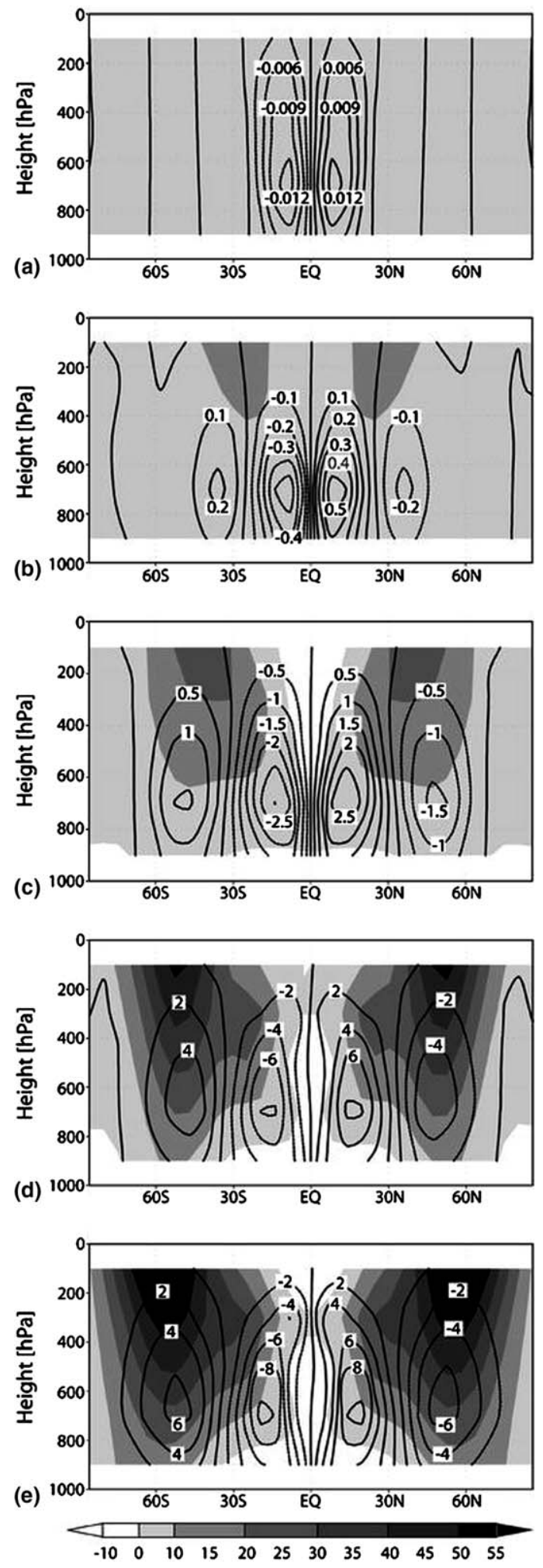

Fig. 4. Zonal mean zonal speed in $\mathrm{m} \mathrm{s}^{-1}$ (shaded contours) and mass stream function in $1010 \mathrm{~kg} \mathrm{~s}^{-1}$ (lines) for (a) $\Delta T_{\mathrm{R}}=20 \mathrm{~K}$, (b) $30 \mathrm{~K}$, (c) $60 \mathrm{~K}$, (d) $130 \mathrm{~K}$ and (e) $190 \mathrm{~K}$ (reprinted from [20] with permission).

In a similar way, the radiative balance of the cold surface is given by

$q+q_{\mathrm{sL}}=q_{\mathrm{L}}$
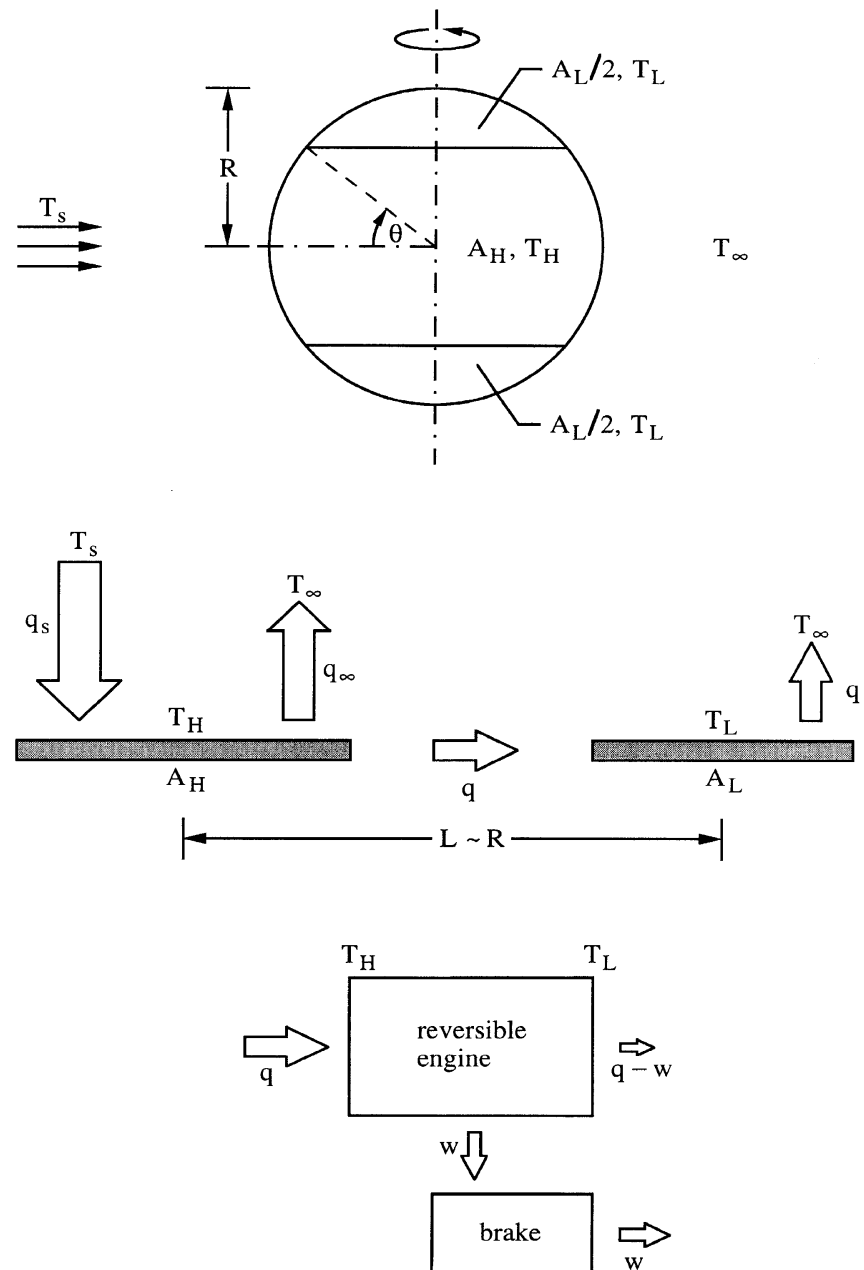

Fig. 5. Earth model with equatorial $\left(A_{\mathrm{H}}\right)$ and polar $\left(A_{\mathrm{L}}\right)$ surfaces, and convective heat current between them.

where $q_{\mathrm{sL}}$ and $q_{\mathrm{L}}$ are the solar radiation absorbed and the heat radiated by the cold surface, respectively,

$q_{\mathrm{sL}}=A_{\mathrm{Lp}}(1-\alpha) f \sigma T_{\mathrm{s}}^{4}$
$q_{\mathrm{L}}=A_{\mathrm{L}}(1-\gamma) \sigma T_{\mathrm{L}}^{4}$

Eqs. (3) and (8) have been simplified by neglecting $T_{\infty}^{4}$ in favor of $T_{\mathrm{L}}^{4}$ and $T_{\mathrm{H}}^{4}$ where $T_{\infty}=3 \mathrm{~K}$ is the temperature of the background. By combining Eqs. (1)-(8) with the notation $x=A_{\mathrm{H}} / A=\sin \theta$, we obtain

$B\left[\arcsin x+x\left(1-x^{2}\right)^{1 / 2}\right]-x T_{\mathrm{H}}^{4}-q /\left[4 \pi R^{2} \sigma(1-\gamma)\right]=0$

and

$B\left[\pi / 2-\arcsin x-x\left(1-x^{2}\right)^{1 / 2}\right]-(1-x) T_{\mathrm{L}}^{4}$

$$
+q /\left[4 \pi R^{2} \sigma(1-\gamma)\right]=0
$$

where $B$ is nearly constant,

$B=T_{\mathrm{s}}^{4} \frac{f}{2 \pi} \frac{1-\alpha}{1-\gamma} \cong 4.1 \times 10^{9} \mathrm{~K}^{4}$ 
$\mathrm{q}$

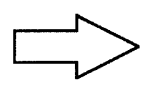

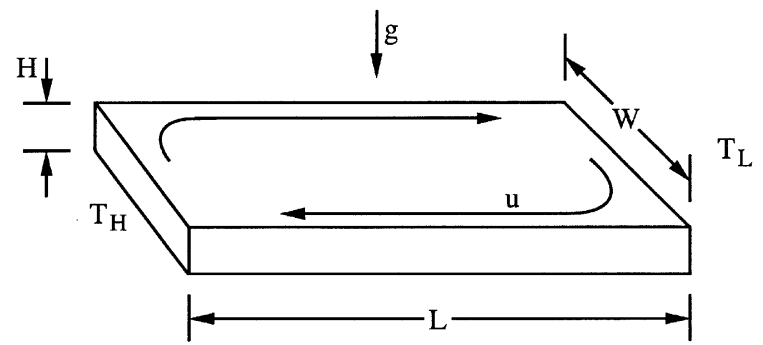

(a)

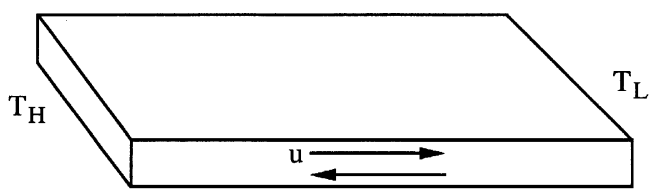

(b)

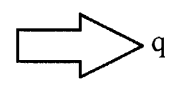

Fig. 6. Natural convection loops in a fluid layer connecting the equatorial and polar surfaces.

The heat current $q$ is driven from $T_{\mathrm{H}}$ to $T_{\mathrm{L}}$ by the buoyancy effect in the layer of fluid that covers the earth's surface. The fluid layer covers an area of flow length $L$ $(\sim R)$ and width $W(\sim R)$, where $R$ is the earth's radius. The vertical length scale of the fluid layer, $H$, will be defined shortly. The length $L$ bridges the gap between $T_{\mathrm{H}}$ and $T_{\mathrm{L}}$.

At the $T_{\mathrm{H}}$-end of the fluid layer, the hydrostatic pressure at the bottom of the layer is $\rho_{\mathrm{H}} g H$. Similarly, at the $T_{\mathrm{L}}$-end the pressure is $\rho_{\mathrm{L}} g H$. The pressure difference in the $L$ direction is

$\Delta P \sim\left(\rho_{\mathrm{L}}-\rho_{\mathrm{H}}\right) g H \sim \rho \beta\left(T_{\mathrm{H}}-T_{\mathrm{L}}\right) g H \xi$

where $\rho$ is the mean fluid density, $\beta$ is the coefficient of volumetric thermal expansion and $\xi=\left(1-\beta g H / R_{\mathrm{g}}\right)^{-1}$ is a factor that results from the expansion and linear approximation of $\rho=P /\left(R_{\mathrm{g}} T\right)$, namely, $\rho_{\mathrm{L}}-\rho_{\mathrm{H}}=\beta \rho\left(T_{\mathrm{H}}-T_{\mathrm{L}}\right)+$ $k_{\mathrm{T}} \rho\left(P_{\mathrm{L}}-P_{\mathrm{H}}\right)$, where $k_{\mathrm{T}}$ is the isothermal compressibility, $k_{\mathrm{T}}=\rho^{-1}(\partial \rho / \partial P)_{T} ;(\xi=1$ for the ocean $)$.

The fluid-layer control volume is exposed to the force $\triangle P W H$ in the $L$ direction. This force is opposed by the shear force felt by the moving fluid over the surface $L W$,

$\Delta P W H=\tau L W$

The average Reynolds shear stress due to the fluctuating component $\left(u^{\prime}\right)$ of speed $(u)$ is

$\tau \sim \overline{\rho\left(u^{\prime}\right)^{2}}$

It is known that in the atmospheric boundary layer the average turbulence intensities $\left[\overline{\left(u^{\prime}\right)^{2}}\right]^{1 / 2}$ are of the order of $0.1[28$, p. 118$]$ and therefore

$\tau \sim 0.01 \rho u^{2}$

By eliminating $\Delta P$ and $u$ between Eqs. (12)-(15) we obtain the horizontal velocity scale

$u \sim 10\left[\beta g \xi\left(T_{\mathrm{H}}-T_{\mathrm{L}}\right) \frac{H^{2}}{L}\right]^{1 / 2}$
The convective heat transfer rate associated with the counterflow between $T_{\mathrm{H}}$ and $T_{\mathrm{L}}$ depends on whether the two branches of the counterflow are in intimate thermal contact. They are not if, for example, the circulation is in the plane $L \times W$, as in the case of $R$-scale oceanic and atmospheric currents that complete loops over large portions of the globe (Fig. 6a). Another example is when the loop is in a vertical plane aligned with the meridian, when the branches of the counterflow are far enough apart and do not exchange heat in a significant way in the vertical direction (Fig. 6b). In such cases the convective heat current is

$q \sim u H W \Lambda c_{p}\left(\rho_{\mathrm{H}} T_{\mathrm{H}}-\rho_{\mathrm{L}} T_{\mathrm{L}}\right)=u H W \Delta P \Lambda c_{p} / R_{\mathrm{g}}$

where we used $P=\rho R_{\mathrm{g}} T$, and $\Lambda$ is the ratio between the total enthalpy change of the atmosphere (sensible heat plus latent heat) and the part corresponding to sensible heat alone. In view of the magnitude of the sources contributing to the atmospheric energy budget $[17$, p. 41$]$, we estimate $\Lambda \sim 1.2$. After using Eqs. (12) and (16), we obtain from Eq. (17)

$q \sim 12 \rho\left(c_{p} / R_{\mathrm{g}}\right)(g \beta \xi)^{3 / 2} H^{3} L^{-1 / 2} W\left(T_{\mathrm{H}}-T_{\mathrm{L}}\right)^{3 / 2}$

The convective current is proportional to $\left(T_{\mathrm{H}}-T_{\mathrm{L}}\right)^{3 / 2}$, not to $\left(T_{\mathrm{H}}-T_{\mathrm{L}}\right)$, as it might have been assumed based on a simple invocation of convection as a mechanism [e.g., Eq. (19) below]. To verify that Eq. (18) provides a realistic estimate of the $q$ scale, note that in the geophysics literature [11] the group

$D \sim \frac{q}{2 \pi R^{2}\left(T_{\mathrm{H}}-T_{\mathrm{L}}\right)}$

is known as the convective conductance in the horizontal direction, expressed per unit of horizontal (earth) area. Note that the proportionality between heat current and temperature difference in the $D$ definition is assumed, not deduced. The value of $D$ was found empirically [13] to be of the order of $0.6-1.1 \mathrm{~W} /\left(\mathrm{m}^{2} \mathrm{~K}\right)$. The theoretical estimate (19) anticipates 
$D \sim 12 \rho\left(c_{p} / R_{\mathrm{g}}\right)(g \beta \xi)^{3 / 2} H^{3} L^{-1 / 2} W R^{-2}(2 \pi)^{-1}\left(T_{\mathrm{H}}-T_{\mathrm{L}}\right)^{1 / 2}$

which for $L \sim \pi R / 4, W \sim \pi R, R \sim 6600 \mathrm{~km},\left(T_{\mathrm{H}}-T_{\mathrm{L}}\right) \sim$ $30 \mathrm{~K}$, and air properties evaluated at $0{ }^{\circ} \mathrm{C}$ yields

$D \sim 7.8 \times 10^{-2}\left(1-\frac{0.13 H}{\mathrm{~km}}\right)^{-3 / 2}\left(\frac{H}{\mathrm{~km}}\right)^{3} \frac{\mathrm{W}}{\mathrm{m}^{2} \mathrm{~K}}$

This estimate agrees with the average empirical value of $D \sim 0.85 \mathrm{~W} /\left(\mathrm{m}^{2} \mathrm{~K}\right)$ when $H$ is of the order of $2 \mathrm{~km}$, which is a realistic transversal length scale for flows in the atmosphere.

Performing the corresponding calculation for the ocean with the average shear stress, we obtain

$\tau \sim \rho \varepsilon_{M} \frac{u}{H}$

where $\varepsilon_{M}$ is the eddy diffusivity for momentum, and $u$ is the velocity component in the $L$ direction. For the order of magnitude of $\varepsilon_{\mathrm{M}}$ we use Prandtl's mixing length model (e.g., Ref. [26, p. 236]), in which we take $H$ to represent the mixing length,

$\varepsilon_{M}=H^{2} \frac{u}{H}=H u$

In other words, $H$ is the vertical dimension of the fluid system that mixes (transfers momentum vertically) while moving horizontally. Note that $H$ is not the vertical extent of the fluid layer.

By eliminating $\Delta P, \tau$ and $\varepsilon_{\mathrm{M}}$ between Eqs. (12) (with $\xi \sim 1$, because $k_{\mathrm{T} \text {,water }} \sim 0 \mathrm{~Pa}^{-1}$ ), (13), (22) and (23) we obtain the horizontal velocity scale

$u \sim\left[\beta g\left(T_{\mathrm{H}}-T_{\mathrm{L}}\right) H^{2} / L\right]^{1 / 2}$

The horizontal convective heat current in the oceanic mixing layer is

$q \sim \rho u H W c_{p}\left(T_{\mathrm{H}}-T_{\mathrm{L}}\right)$

and the corresponding convective conductance in the horizontal direction (with $L \sim \pi R / 4, W \sim \pi R$ ) is

$D \sim \rho c_{p}(g \beta)^{1 / 2} \pi^{-1 / 2} H^{2} R^{-3 / 2}\left(T_{\mathrm{H}}-T_{\mathrm{L}}\right)^{1 / 2}$

By using the properties of water at $10{ }^{\circ} \mathrm{C}$, Eq. (26) becomes:

$D \sim 27\left(\frac{H}{\mathrm{~km}}\right)^{2} \frac{\mathrm{W}}{\mathrm{m}^{2} \mathrm{~K}}$

This shows that $D$ matches $0.85 \mathrm{~W} /\left(\mathrm{m}^{2} \mathrm{~K}\right)$ when $H$ is of order $170 \mathrm{~m}$ : this length scale is compatible with the thickness of mixing layers in the ocean.

Consider now the alternative where the two branches of the counterflow are in good thermal contact. If the counterflow is a loop in the vertical plane $L \times H$, with the warm branch flowing from $T_{\mathrm{H}}$ to $T_{\mathrm{L}}$ over the top, and the cold branch returning along the bottom (Fig. 6b), then Eq. (24) continues to hold, but Eqs. (25) and (17) are replaced by

$q \sim \rho u H W \Lambda c_{p} \Delta T$
Here, $\Delta T$ is the top-bottom temperature difference between the two branches of the counterflow, $\Delta T<T_{\mathrm{H}}-T_{\mathrm{L}}$. The temperature of the warm branch decreases from $T_{\mathrm{H}}$ at the warm entrance, to $T_{\mathrm{L}}+\Delta T$ at the cold exit. The enthalpy drop in the horizontal direction is caused by the heat transfer by eddy motion in the vertical direction, between the two branches of the counterflow,

$u H\left(T_{\mathrm{H}}-T_{\mathrm{L}}-\Delta T\right) \sim \varepsilon_{\mathrm{H}} L \frac{\Delta T}{H}$

where $\varepsilon_{\mathrm{H}}$ is the thermal eddy diffusivity. By noting that $\tau=\varepsilon_{\mathrm{M}} u / H$ and $\varepsilon_{\mathrm{H}} \sim \varepsilon_{\mathrm{M}}$, from Eq. (15) we find that $\varepsilon_{\mathrm{H}} \sim 0.01 H u$, such that Eq. (29) reduces to

$\frac{\Delta T}{T_{\mathrm{H}}-T_{\mathrm{L}}} \sim 0.01 \frac{H}{L} \ll 1$

The convection current (18) becomes

$q \sim 0.12 \rho\left(c_{p} / R_{\mathrm{g}}\right)(g \beta \xi)^{3 / 2} H^{4} L^{-3 / 2} W\left(T_{\mathrm{H}}-T_{\mathrm{L}}\right)^{3 / 2}$

$D \sim 0.12 \rho\left(c_{p} / R_{\mathrm{g}}\right)(g \beta \xi)^{3 / 2} H^{4} L^{-3 / 2}(2 \pi)^{-1} R^{-2} W\left(T_{\mathrm{H}}-T_{\mathrm{L}}\right)^{1 / 2}$

These estimates for $q$ and $D$ are less realistic because they are smaller by a factor of $0.01 H / L \ll 1$ than those of Eqs. (18) and (20).

In conclusion, the convection model with counterflow branches that are not in intimate thermal contact is more realistic (see also Ref. [27]). Such a counterflow poses a minimal convective resistance to heat flow in the flow direction. This feature has been encountered before in constructal theory. The same type of counterflow, i.e., the same minimal resistance principle was used earlier in constructal theory in the prediction of Bénard convection [14].

In the following analysis, we retain Eq. (18) because of the test given under Eq. (19). Combining Eq. (18) with Eq. (9), we arrive at

$C_{3 / 2}\left(T_{\mathrm{H}}-T_{\mathrm{L}}\right)^{3 / 2} \sim B\left[\arcsin x+x\left(1-x^{2}\right)^{1 / 2}\right]-x T_{\mathrm{H}}^{4}$

where the theoretical conductance depends on $x$, and consequently on the latitude $\theta$ (which defines the boundary between the hot and cold surfaces), because $x=\sin \theta, L \sim R \theta$ and $W \sim 2 \pi R \cos \theta$,

$C_{3 / 2}=\frac{12 \rho\left(c_{p} / R_{\mathrm{g}}\right)(g \beta \xi)^{3 / 2} H^{3} L(x)^{-1 / 2} W(x)}{4 \pi R^{2}(1-\gamma) \sigma}$

For $L \sim \pi R / 2, W \sim \pi R, R \sim 6600 \mathrm{~km}, H \sim 2 \mathrm{~km}$, and air at $1 \mathrm{~atm}$ and $0{ }^{\circ} \mathrm{C}$, we find that $C_{3 / 2} \sim 2.5 \times 10^{6} \mathrm{~K}^{5 / 2}$.

In summary, the radiation-convection model reduces to Eqs. (9), (10) and (33), which determine the functions $T_{\mathrm{H}}(x)$ and $T_{\mathrm{L}}(x)$. Unlike earlier thermodynamic optimizations [24,25], the only degree of freedom in this configuration is the area fraction $x$. The constants $B$ and $C_{3 / 2}$ are known, although the $C_{3 / 2}$ estimate is not nearly as accurate as B. We may treat the convective conductance $C_{3 / 2}$ as a parameter that accounts for the accuracy of the convection model.

The fact that the temperature difference $\left(T_{\mathrm{H}}-T_{\mathrm{L}}\right)$ participates in the model as $\left(T_{\mathrm{H}}-T_{\mathrm{L}}\right)^{3 / 2}$ is in agreement with 
the Monin-Obukhov similarity theory [28], provided that the vertical length scale is replaced by the Obukhov length,

$L_{\mathrm{O}}=\frac{u_{*}^{3} \rho c_{p}}{k g \beta q^{\prime \prime}}$

where $\mathrm{u}_{*}$ is the friction velocity that is related to $u$ of Eqs. (15) and (16) as $u_{*}=0.1 u, k \sim 0.4$ is the von Karman constant, and $q^{\prime \prime}=q /(W L)$ is the heat transported by the meridional flow of a vertical air column of unit area. Using Eqs. (16) and (18) with $\beta=1 /(273 \mathrm{~K})$ we obtain

$L_{\mathrm{O}} \sim \frac{R_{\mathrm{g}}}{12 \mathrm{k} \beta g}=1.64 \mathrm{~km}$

which is a realistic scale for the friction layer. The assumption $H \sim L_{\mathrm{O}}$ is reasonable because the Obukhov length is the scale where the atmospheric shear effects are dominant. In brief, according to the Monin-Obukhov theory the mean flow and turbulence characteristics of the earth boundary layer depend on only four independent parameters: (i) the height $z$ above the earth surface, (ii) the friction velocity $u_{*}=(\tau / \rho)^{1 / 2}$ (cf. Ref. [28, p. 144]), (iii) the surface kinematic heat flux $q^{\prime \prime} /\left(\rho \mathrm{c}_{p}\right)$, and (iv) the buoyancy effect $g / T$ (cf. Ref. [28, p. 157]).

Buckingham's $\pi$ theorem states that the only dimensionless combination of these four variables consists of $\zeta=$ $z / L_{\mathrm{O}}$ where $L_{\mathrm{O}}$ is the group shown in Eq. (36). In the analysis presented in this section we arrived at the same conclusion in a different way. The key assumption was made in Eq. (13), where the effective vertical length scale $(H)$ was taken to be the same as the thickness of the zone dominated by shear.

\section{Maximization of global long-term performance}

The earth model with natural convection loops allows us to estimate several quantities that characterize the global performance of atmospheric and oceanic circulation. We pursue this from the constructal point of view, which is that the circulation itself represents a flow geometry that is the result of the maximization of global performance subject to global constraints. The global circulation is driven by the latitudinal temperature gradient, and its objective is to transport heat from the equatorial zone to the polar zones. In order to analyze how this process can be optimized on the basis of the constructal law we begin by considering a conceptual partition of earth surface, the surface fraction $x$ corresponding to the equatorial zone, and the surface fraction $(1-x)$ for the polar zones. The energy balance is represented by Eqs. (9) and (10), which correspond to the equatorial and the polar zones, respectively. Eq. (9) shows the heat current $q$ leaving the equatorial zone (the heat source) as function of $x$ (or latitude $\theta$, because $x=\sin \theta$ ) and average temperature $T_{\mathrm{H}}$, namely $q=q\left(x, T_{\mathrm{H}}\right)$. To each temperature $T_{\mathrm{H}}$ corresponds an infinity of partitions $(x)$ of the surface. The constructal law requires that at each temperature $T_{\mathrm{H}}$ there is an optimal $x$ that maximizes the heat current that leaves the heat source, $\left(\frac{\partial q}{\partial x}\right)_{T_{\mathrm{H}}}=0, \quad\left(\frac{\partial^{2} q}{\partial x^{2}}\right)_{T_{\mathrm{H}}}<0$

In a similar way, the constructal principle requires that there exists a value of $x$ that maximizes the heat current $q\left(x, T_{\mathrm{L}}\right)$, Eq. (10), which enters the heat sink of temperature $T_{\mathrm{L}}$,

$\left(\frac{\partial q}{\partial x}\right)_{T_{\mathrm{L}}}=0, \quad\left(\frac{\partial^{2} q}{\partial x^{2}}\right)_{T_{\mathrm{L}}}<0$

Eqs. (37) and (38) must hold during the optimization of both currents. This additional condition has a simple mathematical expression, as we show next. According to Eq. (10), $T_{\mathrm{L}}$ is a function of $x$ and $q$, while according to Eq. (9) $q$ is a function of $T_{\mathrm{H}}$ and $\mathrm{x}$. Therefore we have the functional relationship

$T_{\mathrm{L}}=T_{\mathrm{L}}\left[x, q\left(T_{\mathrm{H}}, x\right)\right]$

or

$\frac{\partial T_{\mathrm{L}}}{\partial x}=\left(\frac{\partial T_{\mathrm{L}}}{\partial x}\right)_{q}+\left(\frac{\partial T_{\mathrm{L}}}{\partial q}\right)_{x}\left(\frac{\partial q}{\partial x}\right)_{T_{\mathrm{H}}}$

The first derivative in Eq. (38) can also be written as

$\left(\frac{\partial q}{\partial x}\right)_{T_{\mathrm{L}}}=-\left(\frac{\partial T_{\mathrm{L}}}{\partial x}\right)_{q} /\left(\frac{\partial T_{\mathrm{L}}}{\partial q}\right)_{x}$

such that Eq. (40) becomes

$\frac{\partial T_{\mathrm{L}}}{\partial x}=\left(\frac{\partial T_{\mathrm{L}}}{\partial q}\right)_{x}\left[\left(\frac{\partial q}{\partial x}\right)_{T_{\mathrm{H}}}-\left(\frac{\partial q}{\partial x}\right)_{T_{\mathrm{L}}}\right]$

Then, when the optimization conditions (37) and (38) hold, from Eq. (42) we obtain

$\frac{\partial T_{\mathrm{L}}}{\partial x}=0$

From Eq. (10) we can evaluate all the derivatives on the right hand side of Eq. (42). Next, we evaluate the second derivative,

$\frac{\partial^{2} T_{\mathrm{L}}}{\partial x^{2}}=16 \pi R^{2} \sigma(1-\gamma) x T_{\mathrm{L}}^{3}\left(T_{\mathrm{H}}^{4}-T_{\mathrm{L}}^{4}\right)$

which is always positive or zero. Therefore, a point of optimal performance is defined in the $\left(q, T_{\mathrm{L}}, x\right)$ space by three conditions:

$\left(\frac{\partial q}{\partial x}\right)_{T_{\mathrm{H}}}=0, \quad \frac{\partial T_{\mathrm{L}}}{\partial x}=0, \quad \frac{\partial^{2} T_{\mathrm{L}}}{\partial x^{2}}>0$

These imply that $T_{\mathrm{L}}$ must have the minimum value possible.

By following an analogous argument, another point of optimal performance in the $\left(q, T_{\mathrm{H}}, x\right)$ space is defined by the conditions

$\left(\frac{\partial q}{\partial x}\right)_{T_{\mathrm{L}}}=0, \quad \frac{\partial T_{\mathrm{H}}}{\partial x}=0, \quad \frac{\partial^{2} T_{\mathrm{H}}}{\partial x^{2}}<0$ 
which means that at this point $T_{\mathrm{H}}$ must have a maximum. Eqs. (45) and (46) represent the application of the constructal principle to the heat current flowing between the equatorial zone (heat source) and the polar zones (heat sinks), the energy balance of which is represented by Eqs. (9) and (10).

In order to determine the optimal performance of this global system we fix the temperature of the equatorial zone $T_{\mathrm{H}}$ in Eq. (9). This gives birth to the family of curves $q(x)_{T_{\mathrm{H}}}$. To each $T_{\mathrm{H}}$ value corresponds a curve with a well-defined maximum (e.g., $q_{\max }$ and $x_{\text {opt }}$ in Fig. 7), and to each maximum corresponds a temperature $T_{\mathrm{L}}$ that is determined by combining Eqs. (9) and (10) in the form

$x T_{\mathrm{H}}^{4}+(1-x) T_{\mathrm{L}}^{4}=\frac{\pi}{2} B$

The point of optimal performance is defined by the minimum $T_{\mathrm{L}}$, as required by the second and third conditions in Eqs. (45). Fig. 7 shows that there is a well-defined $T_{\mathrm{L}}$ minimum at $T_{\mathrm{L}}=275.5 \mathrm{~K}$, which enables the determination of the point of optimum performance as $x=0.434$, $T_{\mathrm{H}}=293.5 \mathrm{~K}$ and $T_{\mathrm{L}}=275.5 \mathrm{~K}$.

Similarly, we fix $T_{\mathrm{L}}$ in Eq. (10), determine the points $\left(q_{\max }, x_{\mathrm{opt}}\right)$, and then locate the maximal $T_{\mathrm{H}}$, as required by the second and third conditions in Eq. (46). Some of the resulting curves $q(x) T_{\mathrm{L}}$ are shown in Fig. 8, and the curve $q(x)$ corresponding to the maxima of $q$ as function of $x$ is shown later in Fig. 12.

The point of optimal performance determined by this way is located at $x=0.8, T_{\mathrm{H}}=288 \mathrm{~K}$ and $T_{\mathrm{L}}=265.5 \mathrm{~K}$. The $T_{\mathrm{H}}(x)$ and $T_{\mathrm{L}}(x)$ curves corresponding to the first of conditions (45) and (46) are shown in Fig. 9. This figure shows a well-defined minimum for $T_{\mathrm{L}}$, which corresponds to the first optimal performance point, and a well-defined maximum for $T_{\mathrm{H}}$ corresponding to the second optimal performance point.

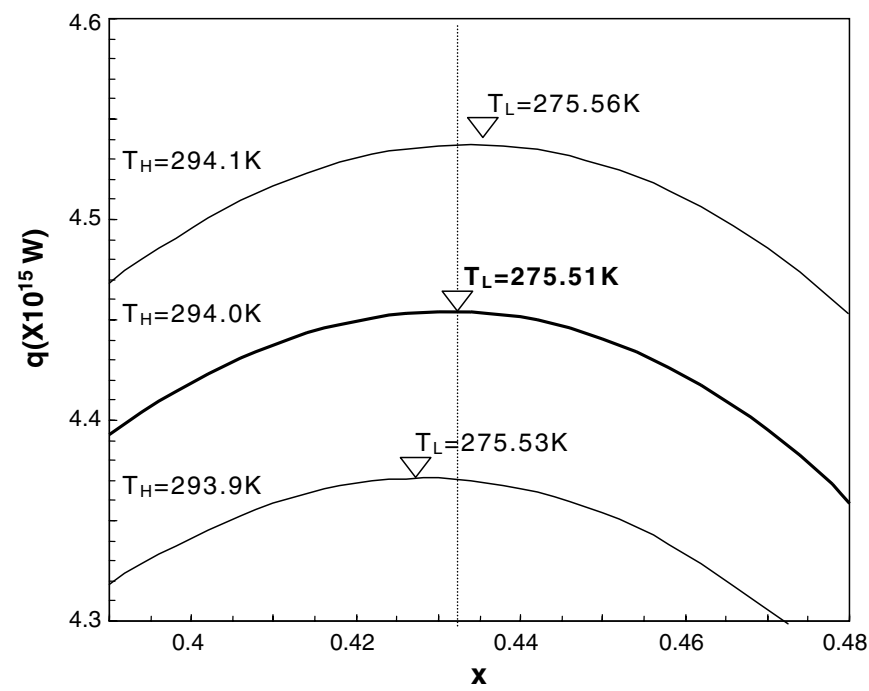

Fig. 7. The heat current $q$ at constant $T_{\mathrm{H}}$, as function of the partitioning of the earth surface $(x)$. Also shown is the $T_{\mathrm{L}}$ temperature corresponding to the maximum of each curve.

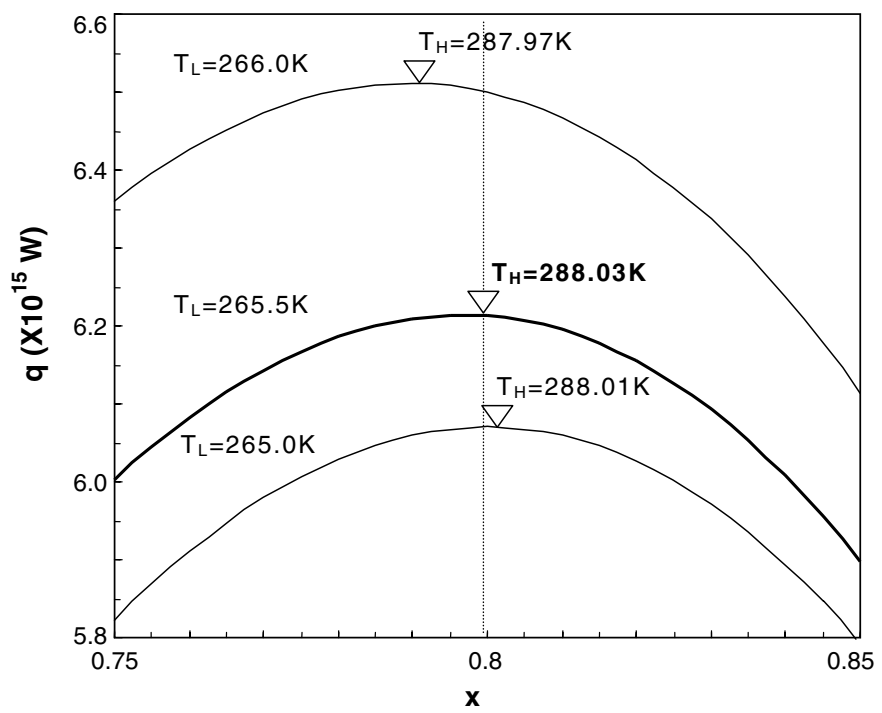

Fig. 8. The heat current $q$ at constant $T_{\mathrm{L}}$, as function of the partitioning of the earth surface $(x)$. Also shown is the $T_{\mathrm{H}}$ temperature corresponding to the maximum of each curve.

The latitudes $\theta$ that correspond to the first and second optimal performance points are $25^{\circ} 40^{\prime}$ and $53^{\circ} 10^{\prime}$ respectively. Fig. 10 shows the two partitions of the earth surface that correspond to these two points. In Case I, the heat source is the equatorial zone of average temperature $T_{\mathrm{H}}=293.5 \mathrm{~K}$, which is located between $25^{\circ} 40^{\prime} \mathrm{N}$ and $25^{\circ} 40^{\prime} \mathrm{S}$. The heat sinks are two surfaces of average temperature $T_{\mathrm{L}}=275.5 \mathrm{~K}$, which are located above the latitude $25^{\circ} 40^{\prime}$. In Case II, the heat sinks are the polar caps above $53^{\circ} 10^{\prime}$, with the temperature $T_{\mathrm{L}}=265.5 \mathrm{~K}$, and the heat source is the equatorial zone of temperature $T_{\mathrm{H}}=288 \mathrm{~K}$. The zones between the latitudes $25^{\circ} 40^{\prime}$ and $53^{\circ} 10^{\prime}$ contribute

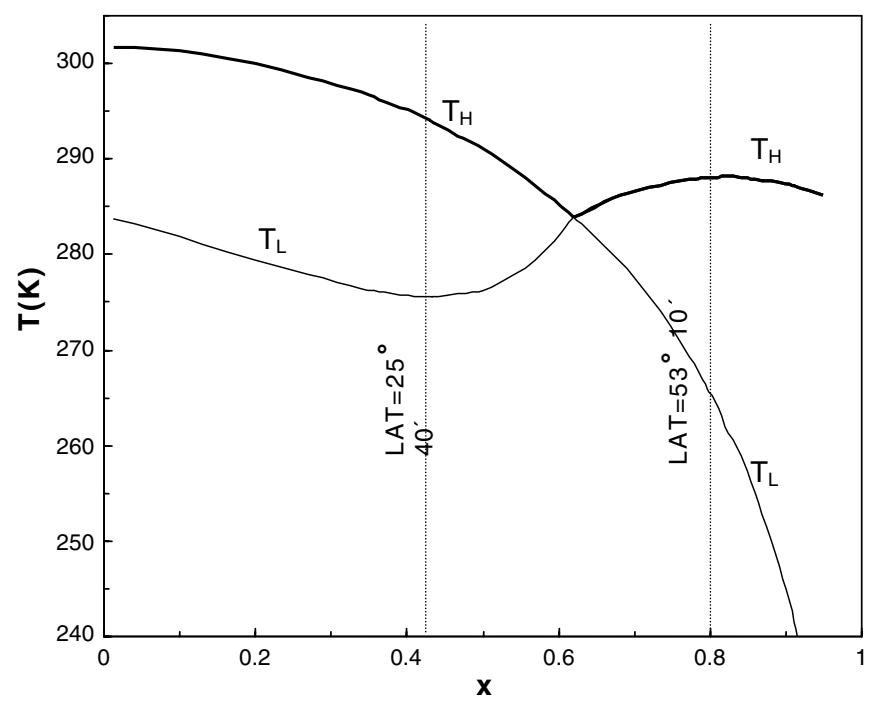

Fig. 9. The temperatures $T_{\mathrm{H}}$ and $T_{\mathrm{L}}$ that maximize the heat current leaving the heat source (the part to the left of the intersection), and the temperatures that maximize the heat current entering the heat sink (the part to the right of the intersection). 

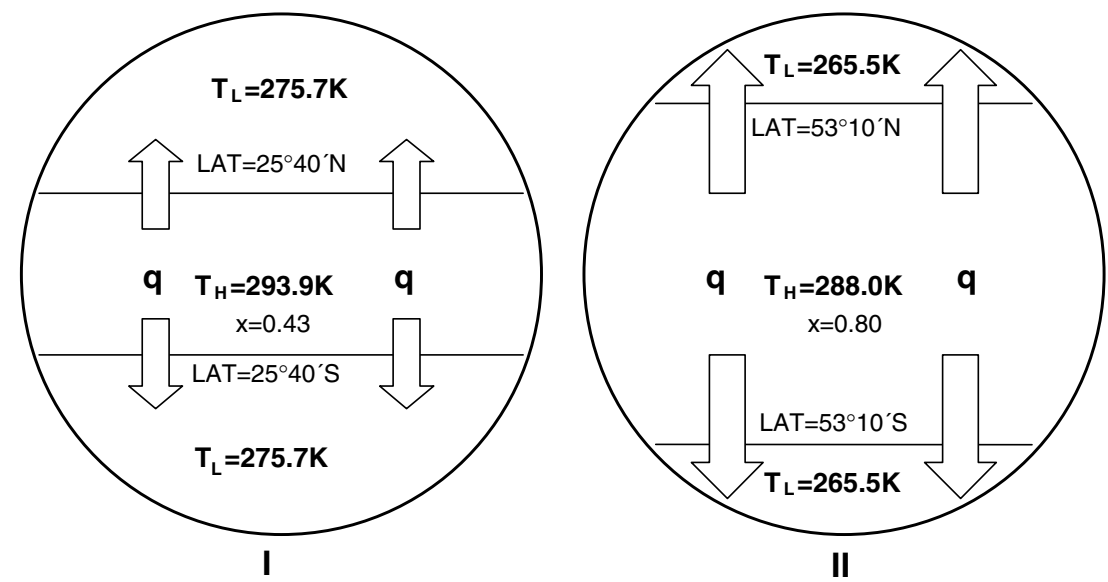

Fig. 10. The two partitionings of the heat surface, which maximize the latitudinal heat current. The surface partitioning $(x)$ is shown in both cases. The corresponding average temperatures of the heat source $\left(T_{\mathrm{H}}\right)$ and heat $\operatorname{sink}\left(T_{\mathrm{L}}\right)$ are also shown.

to the average temperature of the heat source in partition II and to the average temperature of the heat sinks in partition I. In this way the average temperature of this zone $T_{\mathrm{HL}}$ may be evaluated as

$0.434 \times 293.5 \mathrm{~K}+(0.8-0.434) T_{\mathrm{HL}}=0.8 \times 288 \mathrm{~K}$

and its value is $T_{\mathrm{HL}}=281.5 \mathrm{~K}$

The constructal law led to a partition of the earth surface into a heat source between $25^{\circ} 40^{\prime} \mathrm{N}$ and $25^{\circ} 40^{\prime} \mathrm{S}$, and two heat sinks located in the polar caps bounded by the latitudes $53^{\circ} 10^{\prime} \mathrm{N}$ and $\mathrm{S}$, this in order for the system to achieve optimal performance with respect to the meridional heat transport. Between the heat source and sinks, in each hemisphere there is a third surface that participates in the heat transfer process and has an average temperature of $281.5 \mathrm{~K}$. These intermediate surfaces correspond to vertical circulating loops, as we will see when the average temperatures defining this partitioning of surfaces are included in the calculation of the flow variables of the model developed in the preceding section.

It is worth noting that the latitude $25^{\circ} 40^{\prime}$ corresponds very accurately to the boundary between the Hadley and Ferrel cells. Furthermore, the latitude $53^{\circ} 10^{\prime}$ is close to the latitude $60^{\circ}$, which is recognized as the boundary between the Ferrel and the polar cells that represent the mean meridional atmospheric circulation on earth. In Fig. 11 we can observe the cells corresponding to the long-term latitudinal circulation: the Hadley cells, which start at the Equator and go close to $25^{\circ}$, the Polar cells that develop between each pole and latitude $60^{\circ}$, and the Ferrel cells located between the Hadley and the polar cells. These cells are considered to drive the Ferrel cells, which develop between them [29].

It is a remarkable coincidence that the invocation of the constructal law predicts these latitudes as the optimal partitioning of the earth surface with respect to the heat flow along the meridian. We believe that the equator-pole temperature difference plays the major role in the definition of the three-cell regime of meridional circulation in line with the results of Stenzel and von Storch [20]. At slow rotation, the role of the rotation rate of the earth is determinant because it reduces the temperature gradient between the dark and illuminated hemispheres, but for daily rotation periods shorter than $24 \mathrm{~h}$ the rotation rate does not affect significantly the position of the three cells, as shown by Jenkins [19].

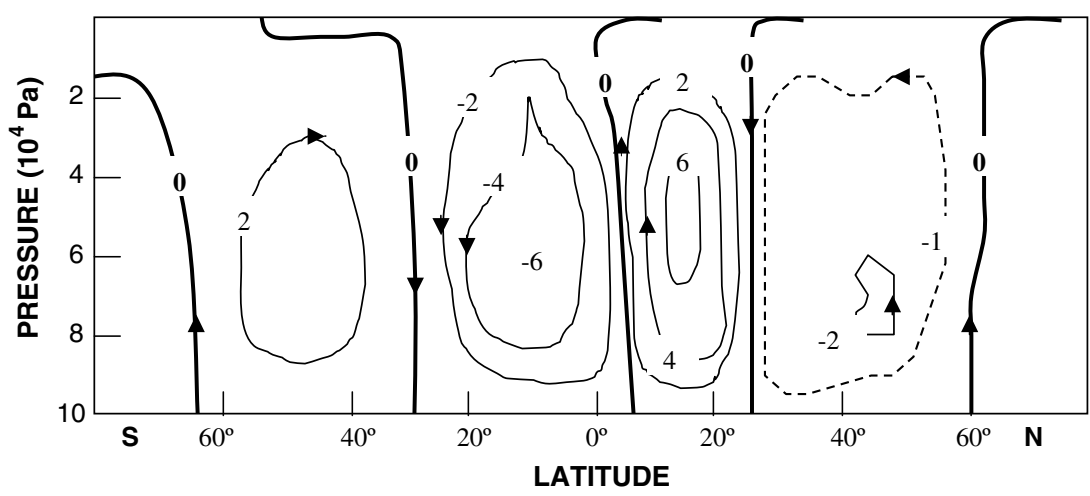

Fig. 11. The atmospheric latitudinal circulation shown for the annual mean. The contour interval is 10 sverdrups $\left(1\right.$ sverdrup $\left.=10^{6} \mathrm{~m}^{3} \mathrm{~s}^{-1}\right)$ and the ordinate corresponds to the height above earth surface as measured in atmospheric pressure units (adapted from [15]). 
The convective conductance in the horizontal direction along the meridian $(D)$ may also be determined from Eq. (19), by using the values of the heat current at each optimal point (Fig. 12). The values of $D$ corresponding to each point are shown in Table 1: they fall in the interval 0.6$1.1 \mathrm{~W} /\left(\mathrm{m}^{2} \mathrm{~K}\right)$, which is the domain of the empirical values [9].

Another coincidence is that each optimal partitioning of the earth surface, namely $\left(x=0.434, T_{\mathrm{H}}=293.9 \mathrm{~K}\right.$, $\left.T_{\mathrm{L}}=275.5 \mathrm{~K}\right)$ and $\left(x=0.8, T_{\mathrm{H}}=288 \mathrm{~K}, T_{\mathrm{L}}=265.5 \mathrm{~K}\right)$ leads to the same average temperature of the earth surface. The average temperature evaluated for these two cases is $283.5 \mathrm{~K}$ :

$$
\langle T\rangle_{\mathrm{I}}=0.434 \times 293.9 \mathrm{~K}+(1-0.434) \times 275.5=283.5 \mathrm{~K}
$$

$$
\langle T\rangle_{\mathrm{II}}=0.800 \times 288 \mathrm{~K}+(1-0.800) \times 265.5=283.5 \mathrm{~K}
$$

Another interesting coincidence occurs when we calculate the overall entropy generated on the earth's surface, which can be derived by accounting for all incoming and outgoing entropy fluxes (heat flux/temperature). Therefore, by using Eqs. (5)-(8) we obtain:

$S_{\text {gen }}=S_{0}(1-\alpha) \pi R^{2}\left(\frac{x}{T_{\mathrm{H}}}+\frac{(1-x)}{T_{\mathrm{L}}}-\frac{1}{T_{\mathrm{s}}}\right)+q\left(\frac{1}{T_{\mathrm{L}}}-\frac{1}{T_{\mathrm{H}}}\right)$

Here the solar constant $S_{0}=1380 \mathrm{~W} / \mathrm{m}^{2}$ is the solar radiation (power per unit area perpendicular to the sun rays), $\alpha$ is

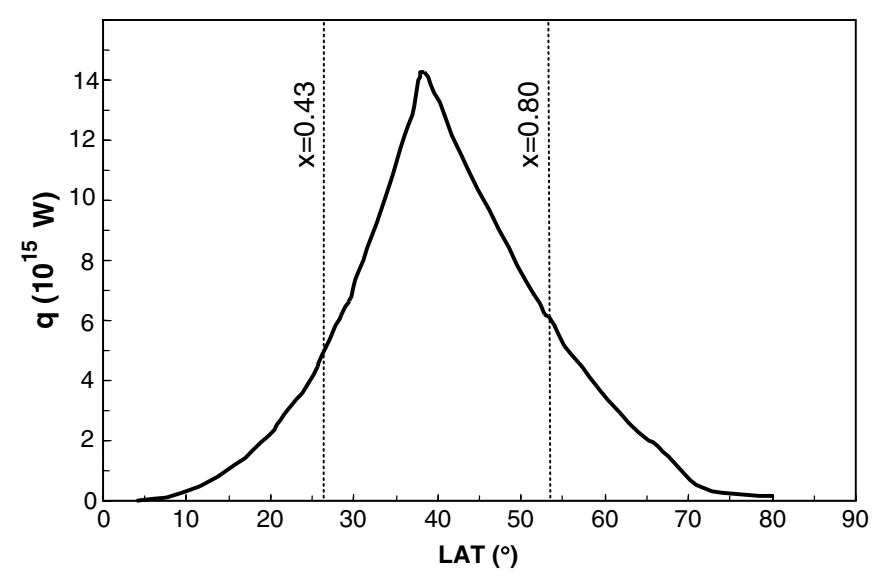

Fig. 12. The heat current from the heat source (left branch) and the heat current to the heat sink (right branch) as function of the latitude. The latitudes at which $q$ is globally maximized are indicated with dashed lines. the albedo of the earth, and $T_{\mathrm{s}}=5762 \mathrm{~K}$ is the sun temperature. The values of the entropy generation corresponding to each optimized partitioning of the earth surface are also shown in Table 1. The coincidence is that the first optimal point (Case I) matches a point of maximum entropy generation, while the second optimal point (Case II) is relatively close to a second entropy generation maximum (Fig. 13). The boundary between the polar and Ferrel cells (latitude $60^{\circ}$ ) falls between the second optimal performance point and the second entropy generation maximum. Moreover, the entropy generation rate has almost the same value in both optimal cases. This agreement between the constructal principle and the principle of maximum entropy generation is a coincidence. There is no sufficient evidence here for deciding in favor or against one principle, however, we cannot associate the second point of maximum entropy generation with any special feature of the latitudinal circulation. However, it is worth noting that the difference between the two entropy generation maxima is less than $1 \%$.

The atmospheric and oceanic circulations transport almost all the latitudinal heat current. The fraction transported by oceanic circulation is not known precisely. In the following analysis we assume that the heat current is transported entirely by atmospheric circulation. The purpose of the analysis is to determine the scales of variables of the latitudinal flow: average latitudinal wind speed $(v)$, average atmospheric pressure difference $\left(P_{\mathrm{L}}-P_{\mathrm{H}}\right)$ and average height of the friction shear layer $(H)$.

A quantity that may be evaluated from this model is the mechanical power that could be generated by a power plant

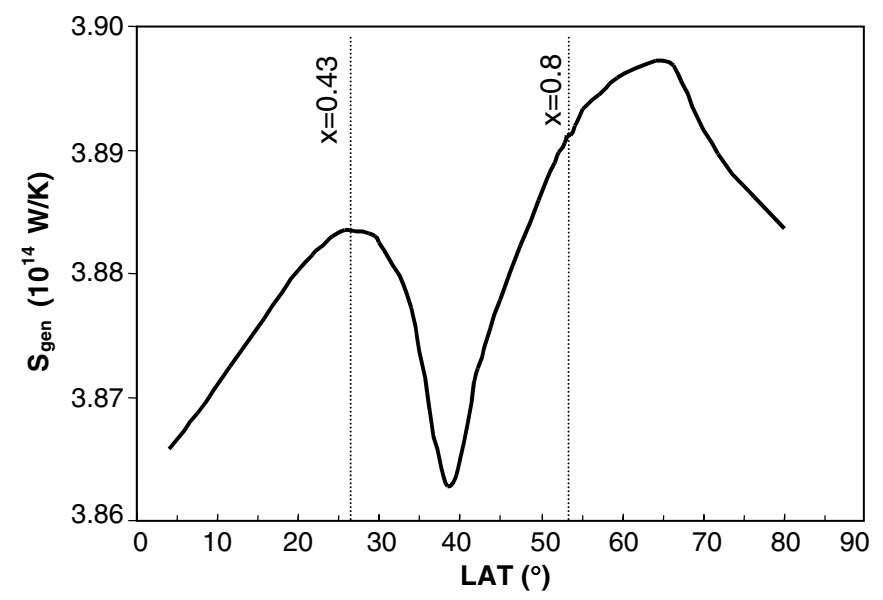

Fig. 13. The overall earth entropy generation as a function of the latitude of the boundary between the heat source and the heat sink.

Table 1

The long-term values of the meridional heat flow between the equatorial and the polar zones that result from the optimization on the basis of the constructal law

\begin{tabular}{llllllll}
\hline & $x$ & Latitude & $T_{\mathrm{H}}(\mathrm{K})$ & $T_{\mathrm{L}}(\mathrm{K})$ & $q(\mathrm{~W})$ & $D\left(\mathrm{~W} / \mathrm{m}^{2}\right)$ & $S_{\text {gen }}(\mathrm{W} / \mathrm{K})$ \\
\hline Case I & 0.434 & $25^{\circ} 40^{\prime}$ & 293.9 & 275.5 & $4.5 \times 10^{15}$ & 0.96 & $3.9 \times 10^{14}$ \\
Case II & 0.8 & $53^{\circ} 10^{\prime}$ & 288 & 265.5 & $6.2 \times 10^{15}$ & 1.06 & $3.9 \times 10^{14}$ \\
\hline
\end{tabular}


operating between $T_{\mathrm{H}}$ and $T_{\mathrm{L}}$, and driven by the heat input $q$. The power output $(w)$ is dissipated by friction in fluid flow (a fluid brake system), and added fully to the heat current $\left(q_{\mathrm{L}}\right)$ that the power plant rejects to $T_{\mathrm{L}}$ (see Fig. 1). The power plant and the fluid brake system occupy the space shown between $A_{\mathrm{H}}$ and $A_{\mathrm{L}}$ in the lower part of Fig. 5. Note the continuity of the heat current $q$ through this composite system. The overall mechanical power is given by

$w=\Delta P u H W$

and, in view of Eq. (17), it is proportional to the heat current

$q=\left(\Lambda c_{p} / R_{\mathrm{g}}\right) w$

In conclusion, the maximization of the mechanical power output is equivalent to the maximization of the heat current from the hot region to the cold region.

Another important concept in this model is the $C_{3 / 2}$ conductivity, which can be determined from Eq. (34). In addition, Eqs. (12), (16) and (18) allow us to calculate the speed of the latitudinal flow (Fig. 14), the height of the friction layer (Fig. 15), and the pressure difference $\left(\Delta P=P_{\mathrm{H}}-P_{\mathrm{L}}\right)$ between the two regions of the earth surface, the heat source $\left(P_{\mathrm{H}}\right)$ and the heat sink $\left(P_{\mathrm{L}}\right)$, see Fig. 16. Table 2 summarizes the results.

The average latitudinal component of the velocity of the air current and the height of the friction layer are higher at the latitude $25^{\circ} 40^{\prime}$ than at the latitude $53^{\circ} 10^{\prime}$ : this is in fair agreement with the observed wind intensity relation between the Ferrel westerlies at each of these latitudes. These winds have a northward component, and start in the vicinity of latitude of $30^{\circ}$, reaching a maximum at the latitude $53^{\circ}$ [29, p. 128].

The pressure in the equatorial zone is $P_{\mathrm{H}} \sim\left(P_{\mathrm{e}}+P_{\theta}\right) / 2$, and in the polar zones is $P_{\mathrm{L}} \sim\left(P_{\theta}+P_{\mathrm{p}}\right) / 2$, where $P_{\mathrm{e}}, P_{\mathrm{p}}$ and $P_{\theta}$ stand for the pressure at the equator, at the pole and at the boundary between the two zones, respectively. By noting that $\Delta P=P_{\mathrm{H}}-P_{\mathrm{L}}=\left(P_{\mathrm{e}}-P_{\mathrm{p}}\right) / 2$, we

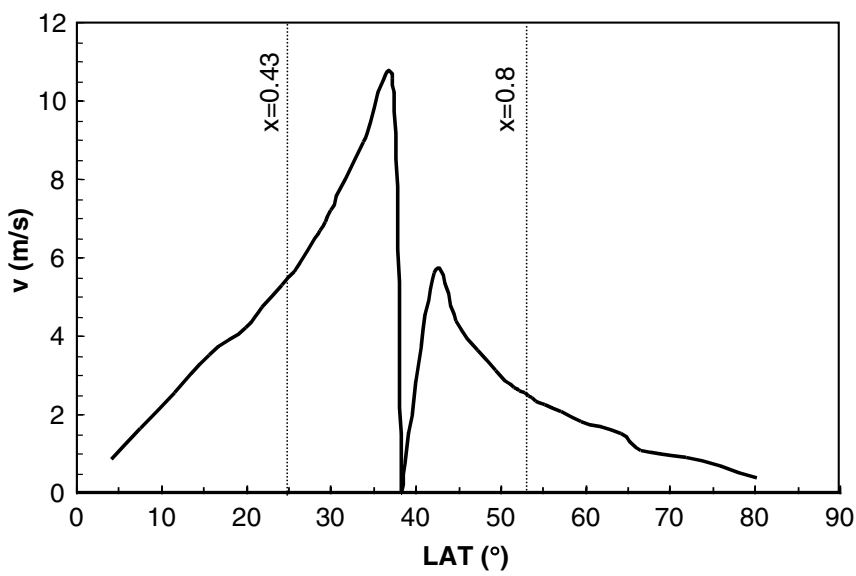

Fig. 14. The average latitudinal wind speed as a function of the latitude of the boundary between the heat source and the heat sink.

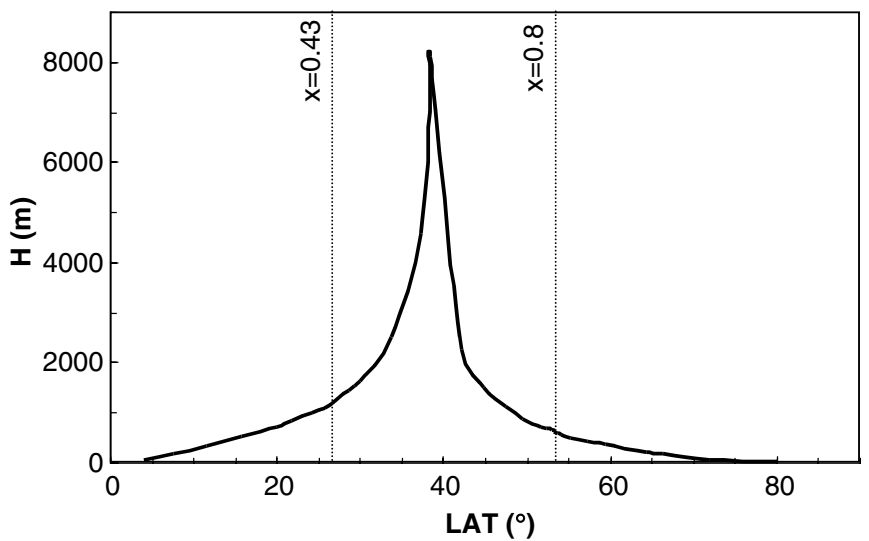

Fig. 15. The average height of the friction layer as a function of the latitude of the boundary between the heat source and the heat sink.

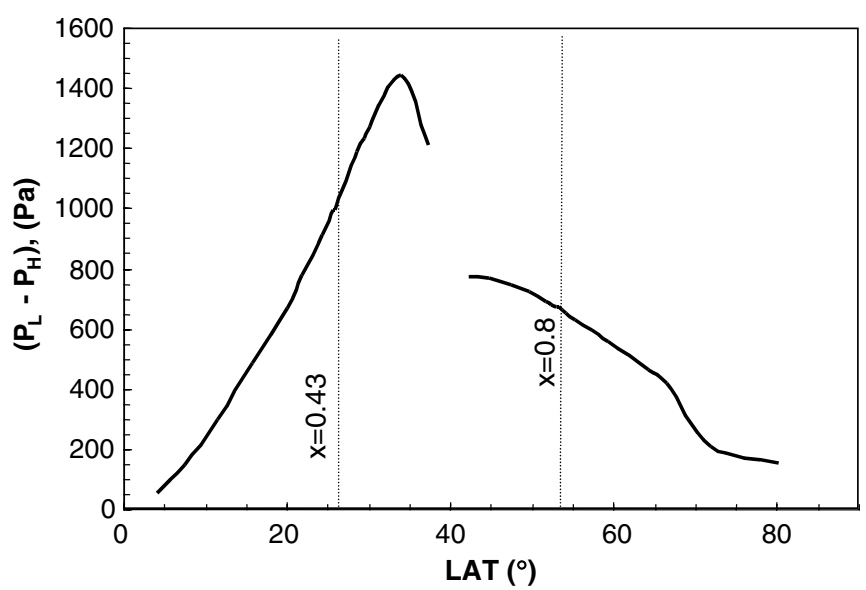

Fig. 16. The average pressure difference between the heat source and the heat sink as a function of the latitude of the boundary between the heat source and the heat sink.

find that the pressure differences between the two zones presented in Table 2 (i.e., $995 \mathrm{~Pa}$ and $672 \mathrm{~Pa}$ ) agree in a scaling sense with the observed average mean sea-level pressure difference between the equator and the poles $\left(P_{\mathrm{e}}-P_{\mathrm{p}}\right)$, which is of the order of $1500 \mathrm{~Pa}$. The mechanical power dissipated by friction, which is determined from Eq. (53), is higher at latitude $25^{\circ} 40^{\prime}$ than at latitude $53^{\circ} 10^{\prime}$, following the result obtained for the heat current.

Another interesting result is that the $C_{3 / 2}$ conductivity has precisely the same value at the latitudes corresponding to the two optimal points (Fig. 17). This is an unexpected result because the $C_{3 / 2}$ conductivity is derived from the flow model with convection loops, while the optimized surface partitioning $(x)$ and the corresponding heat current $(q)$ and temperatures $\left(T_{\mathrm{H}}, T_{\mathrm{L}}\right)$ come from Eqs. (9) and (10), which are quite general and represent energy balances with no relation to a particular model of fluid circulation. If this result is more than a simple coincidence, we may speculate that the heat flow optimization on the basis of the constructal principle which lead to the partitions of the earth surface, is related to a particular form of heat transport 
Table 2

The values of the dynamic variables that correspond to the optimized long-term meridional circulation

\begin{tabular}{lllllll}
\hline$x$ & Latitude & $v\left(\mathrm{~m} \mathrm{~s}^{-1}\right)$ & $H(\mathrm{~m})$ & $\Delta P(\mathrm{~Pa})$ & $w(\mathrm{~W})$ & $C_{3 / 2}\left(\mathrm{~K}^{5 / 2}\right)$ \\
\hline 0.434 & $25^{\circ} 40^{\prime}$ & 5.7 & 1110 & 995 & $1.1 \times 10^{15}$ & $3.04 \times 10^{6}$ \\
0.8 & $53^{\circ} 10^{\prime}$ & 2.5 & 633 & 672 & $1.5 \times 10^{15}$ \\
\hline
\end{tabular}

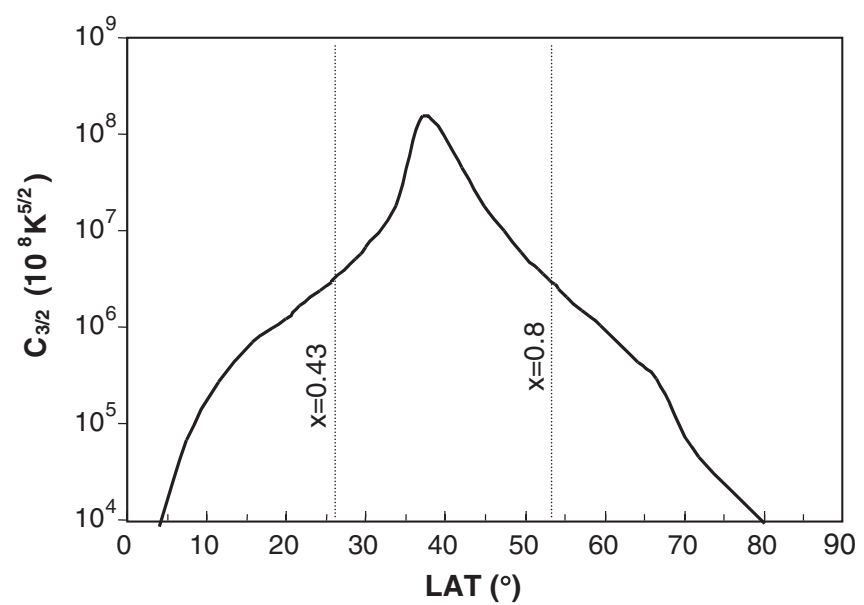

Fig. 17. The conductivity that corresponds to the heat current as a function of the latitude of the boundary between the heat source and the heat sink.

that depends on the $3 / 2$ power of the temperature difference $\left(T_{\mathrm{H}}-T_{\mathrm{L}}\right)$. Furthermore, this particular form of heat transport corresponds to vertical loops in the atmosphere, as shown in Section 2 [see Eqs. (31)-(34)].

In summary, the results reported in this section show that the constructal law provides a basis for understanding and predicting a highly complex flow structure such as the global circulation. In the next section we develop additional results by applying the constructal law at the diurnal scale.

\section{Maximization of performance at the diurnal scale}

At the diurnal scale, the collector area corresponds to the illuminated part of the earth surface, and the radiator corresponds to the dark part. As the sun rays are practically parallel the collector area, the illuminated area is half of the earth surface $\left(x=A_{\mathrm{H}} / A_{\mathrm{L}} \cong 1 / 2\right)$.

The difference between the energy received and emitted by the collector is transported in part by the earth's rotation, while the rest is convected over the earth surface (see Fig. 18). As the boundary between the illuminated and dark surfaces moves with a speed equal to the earth rotation speed at the same latitude, the earth rotation transfers heat continuously from the illuminated region to the dark region even in the absence of convection. This transfer occurs because heat from the earth surface that is heated up during the day crosses continuously the boundary between day and night. We can say that the earth has considerably more freedom than the moon to generate (to morph) its constructal flow configuration, because flu-

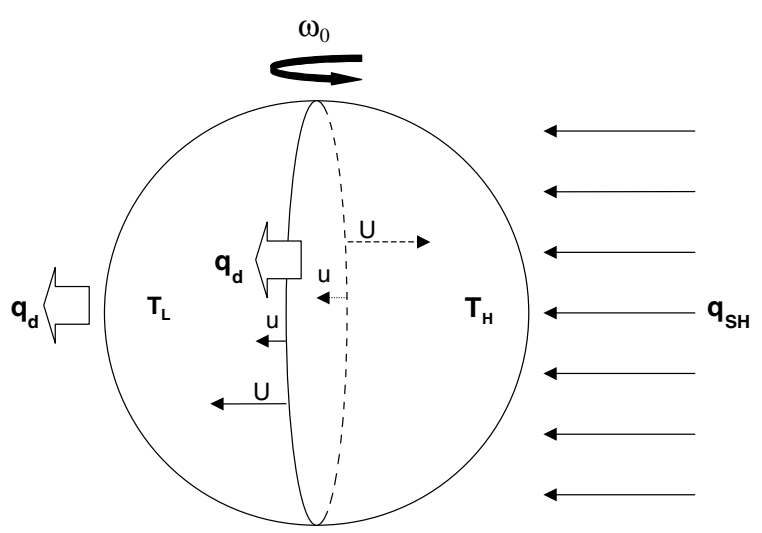

Fig. 18. The heat current $q_{\mathrm{d}}$ between the illuminated and the dark zones on earth. Air from the illuminated zone is entering the dark zone with the velocity component $u$ relative to the earth surface.

ids are free to flow over the earth surface. Because at the diurnal scale the collector area is equal to the radiator area, the parameter that is allowed to vary in the constructal optimization of the flow configuration is the average speed of the atmospheric air relative to the earth surface.

The heat current that flows from the collector at temperature $T_{\mathrm{H}}$ to the radiator at temperature $T_{\mathrm{L}}$ is given by

$q_{\mathrm{d}}=\left(\rho_{\mathrm{H}} c_{p} T_{\mathrm{H}}-\rho_{\mathrm{L}} c_{p} T_{\mathrm{L}}\right)\left[\left(2 U_{0} / \pi\right)+2 u\right] \Lambda \pi R H$

where $U_{0}=462 \mathrm{~m} \mathrm{~s}^{-1}$ is the earth rotation speed at the equator, $H$ is the height of the friction layer, $u$ is the average convection speed of the fluid that represents the motion of the fluid relative to the earth surface (Fig. 18), and $\Lambda \sim 1.2$ has the same meaning as in Eq. (17). The earth rotation speed averaged over the meridian is $U=2 U_{0} / \pi$. With the ideal gas law $P=\rho R_{\mathrm{g}} T$, Eq. (54) becomes

$q_{\mathrm{d}}=\left(P_{\mathrm{H}}-P_{\mathrm{L}}\right) \frac{c_{p}}{R_{\mathrm{g}}}\left(\frac{U_{0}}{\pi}+u\right) \Lambda 2 \pi R H$

The total power dissipated by fluid friction on the earth surface is

$w_{\mathrm{d}}=\left(P_{\mathrm{H}}-P_{\mathrm{L}}\right) u 2 \pi R H$

Eqs. (54)-(56) permit us to express the heat current as

$q_{\mathrm{d}}=w_{\mathrm{d}} \Lambda \frac{c_{p}}{R_{\mathrm{g}}}\left(\frac{U_{0}}{\pi u}+1\right)$

This current is transported to the cold region, and later it is radiated into space,

$q_{\mathrm{d}}=2 \pi R^{2}(1-\gamma) \sigma T_{\mathrm{L}}^{4}$

Note also that Eq. (57) represents the sum of two heat currents: the current that is transported to the radiator by the 
earth rotation in the absence of fluid flow relative to the earth surface,

$q_{d 0}=w_{\mathrm{d}} \Lambda \frac{c_{p}}{R_{\mathrm{g}}} \frac{U_{0}}{\pi u}$

and the current $q_{\mathrm{dc}}$ convected by the fluid motion relative to the earth surface

$q_{\mathrm{dc}}=w_{\mathrm{d}} \Lambda \frac{c_{p}}{R_{\mathrm{g}}}$

The heat current convected by the fluid flow is proportional to the power dissipated in the friction layer, as was the case in the maximization of long-term performance in the preceding section, Eq. (53).

The constructal law requires the maximization of flow access: the fluid flow structure has the purpose of maximizing the heat current $q_{\mathrm{dc}}$ convected from the collector to the radiator. In view of Eq. (60), the maximization of the heat current implies the maximization of the power $w_{\mathrm{d}}$ dissipated by fluid friction. Because the partitioning of the earth surface is fixed $(x=1 / 2)$, the optimization is made with reference to the other free parameters of the flow structure: the speed $u$ and the height of the friction layer, $H$. In what follows, we combine $u$ and $H$ into a non-dimensional group, the Ekman number $(E k)$, with respect to which the convective heat current can be maximized.

Because $U / u \gg 1$, from Eqs. (57) and (58) we obtain

$T_{\mathrm{L}}^{4} \cong C_{0} \frac{\varepsilon_{\mathrm{d}}}{E k}$

where $\varepsilon_{\mathrm{d}}$ is a power density,

$\varepsilon_{\mathrm{d}}=w_{\mathrm{d}} /(2 \pi R H)=\left(P_{\mathrm{H}}-P_{\mathrm{L}}\right) u$

$C_{0}$ is a constant,

$C_{0}=\frac{\Lambda}{4(1-\gamma) \sigma} \frac{c_{p}}{R_{\mathrm{g}}}$

and $E k$ is the Ekman number

$E k=\frac{C_{D} u^{2}}{2 \omega u H}$

The Ekman number represents the strength of the friction forces relative to Coriolis forces, $C_{D} \rho u^{2} / H$ [see Eq. (15), where $\left.C_{D}=0.01\right]$ and $2 \rho \omega u$, respectively. In Eq. (62), $\omega=2 U_{0} /(\pi R)$ represents the average vertical component of the angular velocity along the meridian.

Next, the overall radiative balance is given by Eq. (47) with $A_{\mathrm{H}}=A_{\mathrm{L}}$, or $x=1 / 2$,

$T_{\mathrm{L}}^{4}+T_{\mathrm{H}}^{4}=\pi B$

The average convective speed is given by Eq. (16), which after using Eq. (64) becomes

$T_{\mathrm{H}}=T_{\mathrm{L}}+C_{1} E k^{2}, \quad C_{1}=\frac{16 U_{0}^{2} L}{100 \pi^{2} C_{\mathrm{d}}^{2}\langle\xi\rangle\langle\beta\rangle g R^{2}}$

Factor $C_{1}$ is a constant, $\xi$ has the same meaning as in Eq. (16), and the brackets indicate average values. By combining Eqs. (61), (65) and (66) we obtain

$$
\left[\left(C_{0} \frac{\varepsilon_{\mathrm{d}}}{E k}\right)^{1 / 4}+C_{1} E k^{2}\right]^{4}+C_{0} \frac{\varepsilon_{\mathrm{d}}}{E k}=\pi B
$$

This equation expresses the power intensity $\varepsilon_{\mathrm{d}}$ as function of the Ekman number as shown in Fig. 19. From the curve $\varepsilon_{\mathrm{d}}(E k)$ and Eqs. (61) and (66) we determine $T_{\mathrm{L}}$ and then $T_{\mathrm{H}}$. Next, Eqs. (62) and (64) together with Eq. (12) enable us to calculate the height of the friction layer,

$H=\left(\frac{C_{D} \varepsilon_{\mathrm{d}}}{2 \omega\left(T_{\mathrm{H}}-T_{\mathrm{L}}\right) g\langle\beta\rangle\langle\xi\rangle E k}\right)^{1 / 2}$

The average speed $u$ follows from Eq. (64). In the calculations leading to Eq. (68) we took $L /\langle\xi\rangle \sim 1.2 R$, which results from using $L=\pi^{1 / 2} R$ and $H \sim 1600 \mathrm{~m}$ in the calculation of $\xi$ [see Eq. (12)], in accordance with the scale defined by the Obukhov length [see Eq. (36)].

The convective heat current $q_{\mathrm{dc}}$ is determined from Eqs. (60) and (62). The curve $q_{\mathrm{dc}}(E k)$ is shown in Fig. 20, and has a maximum at $E k=0.2$. The corresponding curves for the fluid flow variables and temperatures are shown as function of $E k$ in Fig. 21. The curve for the pressure difference between the collector and the radiator was determined from Eq. (56).

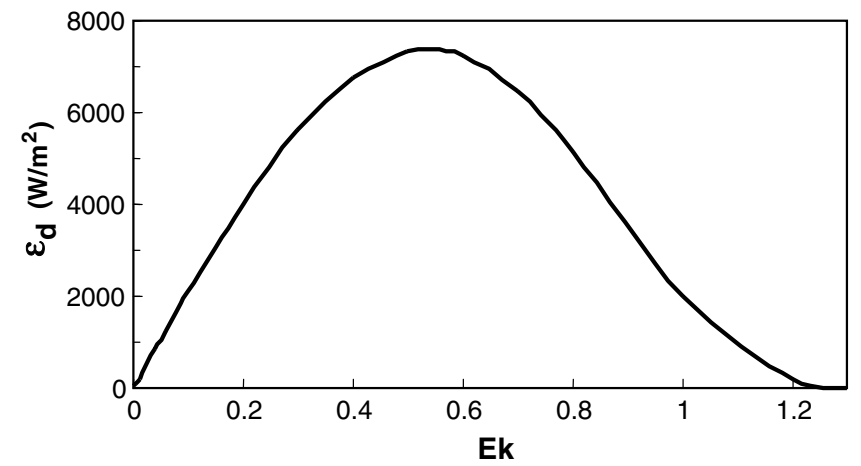

Fig. 19. The average power intensity as a function of the Ekman number.

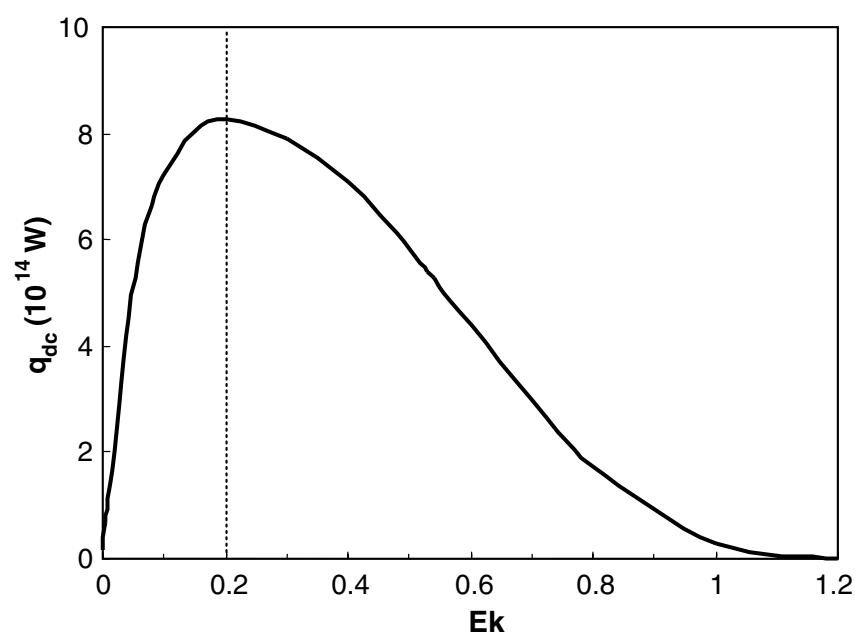

Fig. 20. The convective heat current as a function of the Ekman number. 


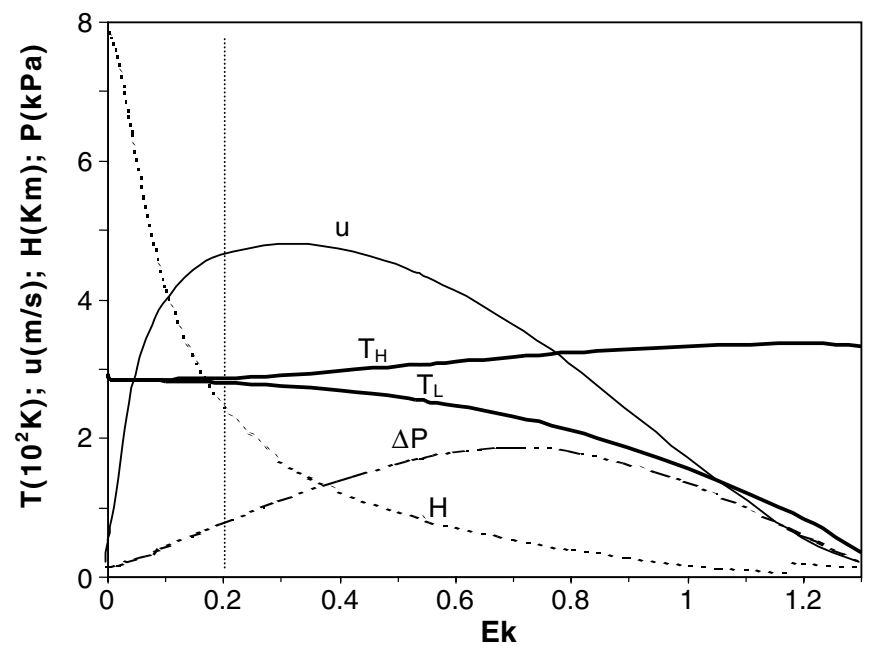

Fig. 21. The temperatures of the illuminated and dark zones, average wind speed, pressure difference, and height of the friction layer, as a function of the Ekman number.

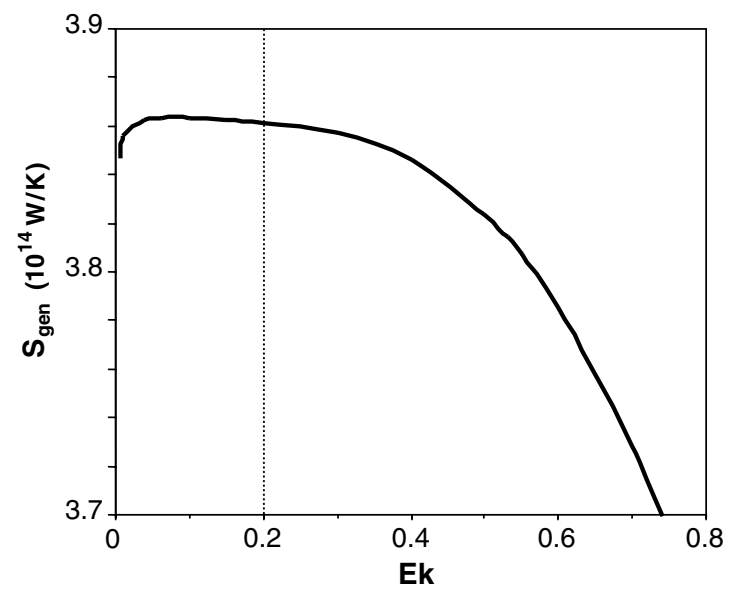

Fig. 22. The overall entropy generation rate as a function of the Ekman number.

The overall entropy generation on earth (Fig. 22) was determined from Eq. (51). In this case, $T_{\mathrm{H}}$ and $T_{\mathrm{L}}$ represent the temperatures of the collector and the radiator, respectively, and $q$ is replaced by $q_{\mathrm{cd}}$. The conductivity $C_{3 / 2}$ has also been determined as function of $E k$, and is reported in Fig. 23.

Optimal performance with respect to heat transfer occurs at $E k=0.2$. Table 3 shows a summary of the flow variables at $E k=0.2$. These results reveal the following important features:

(a) At optimal performance, the average temperature difference $T_{\mathrm{H}}-T_{\mathrm{L}}$ between day and night is of the order of 7 . This agrees with the order of magnitude

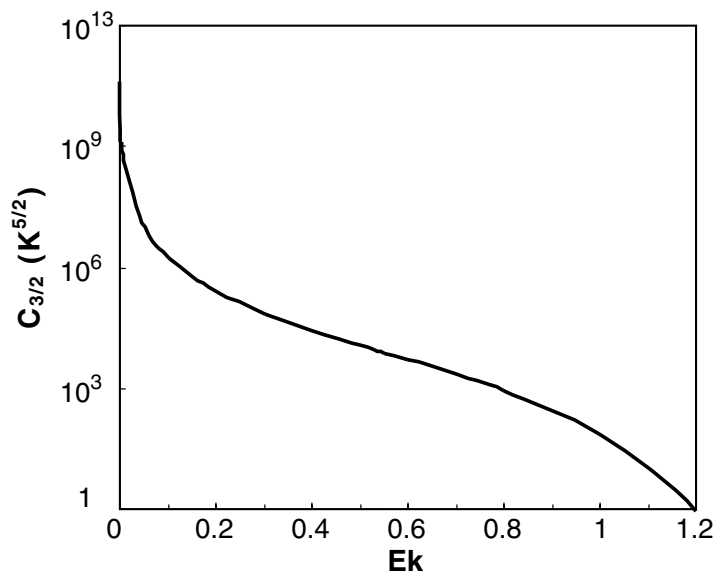

Fig. 23. The conductivity as a function of the Ekman number.

of the average diurnal amplitudes observed on earth, which for the maritime areas is around $3 \mathrm{~K}$ and for continental areas is around $10 \mathrm{~K}[29$, p. 44].

(b) Another important aspect is that the average temperature of the earth surface that results from diurnal optimization is $283.9 \mathrm{~K}$. This is practically the same as the average temperature found based on long-term analysis (283.5 K, Section 3).

(c) The entropy generation rate calculated on the diurnal scale has the same value as $S_{\text {gen }}$ calculated on the long-term scale. The ways in which the variables used in these two $S_{\text {gen }}$ calculations were derived are very different. For the long term, the heat current was determined as function of the earth partition $x$, while for the diurnal scale it was determined as function of $E k$, which involves the earth rotation speed, the height of the friction layer and the fluid flow speed. The maximum of the entropy generation rate is located close to the point of optimum performance, but does not coincide with that point.

(d) The variables defining the properties of the friction layer have values of the same order of magnitude as those determined for the long term. This occurs in spite of the fact that the temperatures $\left(T_{\mathrm{H}}, T_{\mathrm{L}}\right)$ and the heat current differ from the diurnal case to the long-term case.

(e) Dai and Wang [30] used pressure data of the period 1976-1997 from meteorological stations covering the earth's surface and found that diurnal tides between dark and illuminated hemisphere exist in global surface pressure fields, with amplitudes of order $200 \mathrm{~Pa}$. The theoretical results shown in Table 3 predict amplitudes of order $890 \mathrm{~Pa}$. Even though the prediction did not match the observed values, it is of same order.

Table 3

The values of the thermal and dynamic variables that correspond to the optimized diurnal heat transport between the illuminated and dark hemispheres

\begin{tabular}{lllllllll}
\hline$q_{\mathrm{dc}}(\mathrm{W})$ & $T_{\mathrm{H}}(\mathrm{K})$ & $T_{\mathrm{L}}(\mathrm{K})$ & $D\left(\mathrm{~W} / \mathrm{m}^{2}\right)$ & $u\left(\mathrm{~m} \mathrm{~s}^{-1}\right)$ & $H(\mathrm{~m})$ & $\left(P_{\mathrm{L}}-P_{\mathrm{H}}\right)(\mathrm{Pa})$ & $S_{\text {gen }}(\mathrm{W} / \mathrm{K})$ & $C_{3 / 2}\left(\mathrm{~K}^{5 / 2}\right)$ \\
\hline $8.1 \times 10^{14}$ & 287.4 & 280.4 & 0.47 & 4.5 & 2440 & 890 & $3.9 \times 10^{14}$ & $2.5 \times 10^{6}$ \\
\hline
\end{tabular}


(f) The diurnal heat current between the illuminated and dark hemispheres $\left(q_{\mathrm{dc}}\right)$ is roughly 6 or 7 times lower than the long-term poleward current. However, the convective conductance in the horizontal direction $(D)$ and the conductivity $\left(C_{3 / 2}\right)$ have values of the same order as those obtained for the long-term case.

\section{Concluding remarks}

In summary, the optimization of the configuration of the earth circulation system (engine + brake, Figs. 1 and 5 ) on the basis of the constructal law was performed with respect to variables that differ greatly: the earth surface partition $x$ for the long-term poleward transport of heat, and the Ekman number for the geometrical and dynamic variables of the flow at the diurnal scale. The results of both optimization processes agree very well with the observed values. They are also in agreement when values of the same variable are compared after the long-term and diurnal optimizations. Several highly intriguing coincidences have been identified throughout this paper. The accumulation of coincidences between theoretical predictions and natural facts adds weight to the claim that the constructal law is a law of nature [1-4].

Earlier applications of the constructal law $[1,2]$ recommended it as a self-standing law that is distinct from the second law. That the optimized geometric features are consistent with features observed in nature affirms the law status of the principle. It is a thermodynamic law because it completes the thermodynamic description of flow systems under constraints. Flow and dissipation (irreversibility) must be distributed in special ways in space and time.

To see why the constructal law of generation of flow architecture is distinct from the second law, let us review the essence of thermodynamics. Thermodynamic theory was developed in order to account for the functioning of heat engines. The first law accounts for the conservation of energy, and serves as definition of energy as a concept. The second law accounts for the generation of entropy (irreversibility), or the one-way nature of currents that overcome resistances, and serves as definition of entropy as a concept.

The first law and the second law account for the functioning of a given (observed, assumed) heat engine configuration. The history of flow systems, i.e., the evolution of configurations (e.g., heat engines, Ref. [1, p. 374]) shows what the first law and the second law are not covering: the case-by-case increase in performance, in time. Every class of flow structures exhibits this behavior, from river basins to animals and heat engines. Each system in its class represents a flow architecture. New flow systems coexist with old systems, but persist in time if they are better, while older systems gradually disappear. This never-ending parade of flow systems represents the generation of flow architecture - the generation of geometric form as the clash between objective and constraints in flow systems.
Physicists have always been intrigued by the mystery of configuration generation, which is foreign to dynamics. Before the constructal law, no straightforward connection has been envisaged in order to bring together these two aspects (configuration and dynamics) in describing the evolution of the systems in space and in time. Feynman et al. [31, Chapter 19, p. 14] made the following observation, which shows that they were well aware of the deficiency: "Among the principles that I could mention I noticed that most of them sprang in one way or another from the least action principle of mechanics and electrodynamics. But there is also a class that does not. As an example, if currents are made to go through a piece of material obeying Ohm's law, the currents distribute themselves inside the piece so that the rate at which heat is generated is as little as possible. Now, this principle also holds, according to classical theory, in determining even the distribution of the velocities of the electrons inside a metal which is carrying a current."

The thermodynamics of non-equilibrium systems with configuration is an example of how science makes progress. It does, but in steps. The field reached an impasse because the scientists' knowledge and imagination raced well ahead of the concepts and language that are currently accepted. The knowledge and imagination consider "design" (order, optimization maximization, etc.) as evident. The current language is stuck in the concept of 'entropy', disorder and the microscopic.

The word entropy goes not appear in constructal theory. Entropy is the word and concept of the second law of thermodynamics, which it serves very well. The physics covered by the second law is the phenomenon of irreversibility: every thing flows from high to low (there are other equivalent statements [1]). The occurrence of organization (configuration, macroscopic complexity) in nature is an entirely different phenomenon. This phenomenon calls for its own principle and concepts. The constructal law answered this need. The words that appear in the constructal law are the generation of flow configuration, in time.

\section{References}

[1] A. Bejan, Advanced Engineering Thermodynamics, second ed., Wiley, New York, 1997, Chapter 13.

[2] A. Bejan, Shape and Structure, from Engineering to Nature, Cambridge University Press, Cambridge, UK, 2000.

[3] A. Bejan, S. Lorente, The constructal law and the thermodynamics of flow systems with configuration, Int. J. Heat Mass Transfer 47 (2004) 3203-3214.

[4] A. Bejan, S. Lorente, La Loi Constructale, L'Harmattan, Paris, 2005

[5] R. Swenson, Emergent attractors and a law of maximum entropy production: foundations to a theory of general evolution, Syst. Res. 6 (1989) 187-197.

[6] W.V.R. Malkus, The heat transport and spectrum of turbulence, Proc. R. Soc. Ser. A 225 (1954) 196-212.

[7] E.N. Lorenz, Available potential energy and the maintenance of the general circulation, Tellus 7 (1955) 157-167.

[8] G.W. Paltridge, Global dynamics and climate-a system of minimum entropy exchange, Quant. J. R. Met. Soc. 101 (1975) 475-484. 
[9] L.L. Schulman, A theoretical study of the efficiency of the general circulation, J. Atmos. Sci. 34 (1977) 559-580.

[10] G.W. Paltridge, The steady-state format of global climate, Quart. J. R. Met. Soc. 104 (1978) 927-945.

[11] G.R. North, Energy balance climate models, Rev. Geophy. Space Phys. 19 (1981) 91-121.

[12] C.A. Lin, An extremal principle for a one-dimensional climate model, Geophys. Res. Lett. 9 (1982) 716-718.

[13] R.D. Lorenz, J.I. Lunine, C.P. McKay, P.G. Withers, Titan, Mars and Earth: entropy production by latitudinal heat transport, Geophys. Res. Lett. 25 (2001) 415-418.

[14] R.A. Nelson Jr., A. Bejan, Constructal optimization of internal flow geometry in convection, J. Heat Transfer 120 (1998) 357-364.

[15] E.N. Lorenz, Climate predictability, The Physical Basis of Climate and Climate Modeling, World Meteorological Organization, Geneva, Switzerland, GARP, vol. 16, 1975.

[16] J.P. Peixoto, A.H. Oort, Physics of Climate, American Institute of Physics, New York, 1992, pp. 520-1992.

[17] G.A. Mehel, Global Coupled Models: Atmosphere, Ocean, Sea Ice, Climate System Modelling, Cambridge University Press, Cambridge, 1992, pp. 555-81.

[18] A. Navarra, G. Boccaleti, Numerical general circulation experiments of sensitivity to Earth rotation rate, Climate Dyn. 19 (2002) 467-483.

[19] G.S. Jenkins, A sensitivity study of changes in Earth's rotation rate with an atmospheric general circulation model, Global Planet. Change 11 (1996) 141-154.
[20] O.J. Stenzel, J.S. von Storch, On the effect of thermal forcing on the global atmospheric angular momentum and the general circulation, Climate Dyn. 22 (2004) 415-427.

[21] A. Bejan, Advanced Engineering Thermodynamics, Wiley, New York, 1988, pp. 520-522.

[22] J.M. Gordon, Y. Zarmi, Wind energy as a solar-driven heat engine: a thermo-dynamic approach, Am. J. Phys. 57 (1989) 995-998.

[23] A. De Vos, G. Flater, The maximum efficiency of the conversion of solar energy into wind energy, Am. J. Phys. 59 (1991) 751-754.

[24] A. De Vos, Endoreversible Thermodynamics of Solar Energy Conversion, Oxford University Press, Oxford, UK, 1992, pp. 5367.

[25] A. De Vos, P. Van der Wel, The efficiency of the conversion of solar energy into wind energy by means of Hadley cells, Theor. Appl. Climatol. 46 (1993) 193-202.

[26] A. Bejan, Convection Heat Transfer, Wiley, New York, 1984, p. 111.

[27] A. Bejan, A.H. Reis, Thermodynamic optimization of global circulation and climate, Int. J. Energy Res. 29 (2005) 303-316.

[28] S.P. Arya, Introduction to Micrometeorology, Academic Press, London, 1988.

[29] R.G. Barry, R.J. Chorley, Atmosphere, Weather and Climate, seventh ed., Routledge, London, 1998

[30] A. Dai, J. Wang, Diurnal and semidiurnal tides in surface pressure fields, J. Atmos. Sci. 56 (1999) 3874-3891.

[31] R.P. Feynman, R.B. Leighton, M. Sands, The Feynman Lectures on Physics, sixth ed., Addison-Wesley Pub. Co., Reading, MA, 1964. 